“C 2016 IEEE. Personal use of this material is permitted. Permission from IEEE must be obtained for all other uses, in any current or future media, including reprinting/republishing this material for advertising or promotional purposes, creating new collective works, for resale or redistribution to servers or lists, or reuse of any copyrighted component of this work in other works." 


\section{Distributed MIMO Broadcasting: Reverse Compute-and-forward and Signal-space Alignment}

Tao Yang, Member, IEEE,

\begin{abstract}
We study a downlink distributed MIMO system where a central unit (CU) broadcasts messages to $K^{\prime}$ users through $K$ distributed BSs. The $\mathrm{CU}$ is connected to the BSs via $K$ independent rate-constrained fronthaul (FH) links. The distributed BSs collectively serve the users through the air. We propose a new network coding based distributed MIMO broadcasting scheme, using reverse compute-and-forward and signalspace alignment. At the $\mathrm{CU}$, a network coding generator matrix is employed for pre network coding of the users' messages. The network coded messages are forwarded to the BSs, where the FH rate-constraint determines the actual number of network-coded messages forwarded to the BSs. At the BSs, linear precoding matrices are designed to create a number of bins, each containing a bunch of spatial streams with aligned signal-spaces. At each user, post physical-layer network coding is employed to compute linear combinations over the $\mathrm{NC}$ messages w.r.t. the bins, which reverses the pre network coding and recovers the desired messages. We derive an achievable rate of the proposed scheme based on the existence of NC generator matrix, signal-space alignment precoding matrices and nested lattice codes. Improved rate and degrees of freedom over existing interference alignment and compress-and-forward schemes are shown. Numerical results demonstrate the performance improvement, e.g., by as much as $70 \%$ increase in throughput over benchmark schemes.
\end{abstract}

Index Terms-Cloud RAN, CoMP, MIMO, distributed MIMO, broadcast channel, physical-layer network coding, compute-and-forward, interference alignment

\section{INTRODUCTION}

Distributed MIMO (D-MIMO) system finds applications in a wide range of state of the art wireless communication networks, such as coordinated multi-point (CoMP) in LTEadvanced and $5 \mathrm{G}$ cellular networks, local area networks with WiFi coordination, cloud radio access network (C-RAN), heterogeneous network and wireless sensor networks [1] [2] [3] [4] [5]. Advanced interference management techniques can be employed in D-MIMO, e.g., using base station cooperation and multi-cell joint processing, leading to increased spectral efficiency and energy efficiency.

In a generic downlink D-MIMO system, a centralprocessing unit $(\mathrm{CU})$ broadcasts messages to many users through a number of distributed BSs. The $\mathrm{CU}$ is connected to the BSs via independent rate-constrained front-haul (FH) links. The distributed BSs collectively serve the users through the air. The operating of such a system is referred to as distributed MIMO broadcasting. In most practical scenarios, the FH links have limited capacity, which gives rise to a

This work is supported by Australian Research Council (ARC) Discovery early career researcher award (DECRA) with Grant No. DE150100636.

T. Yang is with the Global Big Data Technologies Centre, University of Technology Sydney, Australia (e-mail: tao.yang@uts.edu.au). fundamental impact on the design of the D-MIMO systems for various applications [6] [7] [8].

From the literature, D-MIMO with rate-constrained FH has attract considerable attentions from various aspects. The D-MIMO systems was studied from a CoMP perspective, where coordinated beamforming and joint processing are considered and compared [9] [10]. For the uplink D-MIMO with single-antenna nodes, a simplified Wyner model was considered where cooperative decoding using codebook information and oblivious bases stations are studied in [11] [12]. As inspired by the fact that network coding is required to achieve the multi-cast capacity of the network [13], several network coding and physical-layer network coding based schemes were considered for the uplink D-MIMO. A linear network coding approach based on a pre-assigned network coding generator matrix was proposed for the uplink DMIMO with receiver channel state information (CSI) only, where a full diversity gain is achieved [14]. The computeand-forward (CF) strategy and physical-layer network coding (PNC) techniques can be employed in the uplink D-MIMO system for better utilization of interference [15] [16] [17]. The CF strategy directly generates network coded messages from the superimposed interfering signals, without decoding each individual messages, through structured linear or lattice codes, leading to increased information rate or even degrees of freedom (DoF) gain [18]. For the case with multi-antenna nodes and global CSI, the CF strategy can be further enhanced by incorporating signal-space alignment (SSA) techniques, where considerable degrees of freedom gain can be achieved [19] [20] [21] [22] [23]. For the downlink D-MIMO, oblivious BSs and large system performance analysis are studied in [24]. A network coding based approach called reverse CF was proposed where single-antenna nodes are considered [25]. To date, the ultimate performance and design of downlink DMIMO with rate-constrained FH remains unclear literature. It remains largely unknown on how to realize the benefits of $\mathrm{CF}$ or PNC in the downlink D-MIMO system.

In this paper, we consider a downlink distributed MIMO system where a CU wants to broadcast messages to $K^{\prime}$ users through $K$ distributed BSs. Each of the BSs and user nodes is equipped with multi-antenna. We consider the scenario with moderate FH rate-constraint, i.e., the FH capacity and the information capacity of the air-interface are of the same order.

This paper contributes to this subject through the following aspects. We propose a new distributed MIMO broadcasting scheme, which consists of three key techniques:

Pre network coding: A network coding generator matrix 
is first designed. Based on that, the $\mathrm{CU}$ generates finite field linear combinations of the $K^{\prime}$ users' messages. These linear message-combinations are referred to as network-coded (NC) messages. A subset of NC messages is forwarded to each BS via the FH link. The FH rate-constraint determines the actual number of NC messages that are forwarded to the BSs.

Signal-space alignment: At a BS, each of its transmitted spatial streams carries one NC message. Distributed precoding matrices are designed and employed by the $K$ BSs, such that the signal-spaces of the spatial streams from various BSs are desirably aligned at each user's receiver. The pattern of the signal-space alignment is determined by the choice of the network coding generator matrix.

Post Physical-layer network coding (Reverse CF): At each user node, for each aligned signal-space, a linear combination of the NC messages is computed using PNC. Most importantly, this post PNC operation exactly equalizes (or reverses) the pre network coding at the $\mathrm{CU}$, such that the linear combination of the $\mathrm{NC}$ messages is just the desired message of that user.

Our proposed scheme borrows the idea of reverse CF from [25], with generalization to the scenario with multi-antenna nodes. We show that the pre-network coding matrix and the signal-space alignment precoding matrices are coupled, and investigate the properties and design of these two matrices. We prove the achievable rate of the proposed scheme based on the existence of pre-network coding matrix, nested lattice codes and signal-space alignment precoding matrices.

We also show the achievable DoF of DMIMO with our proposed scheme. We analytically show that considerably improved achievable rate and DoF over existing orthogonal transmission, interference alignment and oblivious base station schemes are obtained. Numerical results are also presented to demonstrate the performance improvement of the proposed scheme, e.g., by as much as 70 percent increase in throughput over benchmark schemes at practical SNR values.

\section{SySTEM MOdeL}

Consider a downlink D-MIMO system where a central unit (CU) wants to broadcast messages to $K^{\prime}$ users through $K$ distributed BSs. The $\mathrm{CU}$ is connected to the $K \mathrm{BSs}$ via $K$ independent rate-constrained front-haul links. The $K$ BSs collectively serve the $K^{\prime}$ users. BS $i$ has $N_{i}$ antennas and User $j$ has $N_{j}^{\prime}$ antennas, $i=1, \cdots, K, j=1, \cdots, K^{\prime}$. The BSs are not mutually connected. The system consists of a front-haul (FH) layer and an air-interface layer, as depicted in Fig. 1. At the FH layer, the CU sends some functions of the users' messages to the distributed BSs via independent FH links, while satisfying the constraint on FH capacity. At the air-interface layer, $K$ BSs distributively broadcast their signals towards the $K^{\prime}$ users through the air.

\section{A. FH Layer}

Let the messages for the $K^{\prime}$ users be denoted by row vectors $\mathbf{b}_{j}, j=1, \cdots, K^{\prime}$. The length of $\mathbf{b}_{j}$ is denoted by $m_{j}$, and the entries of $\mathbf{b}_{j}$ belong to $\operatorname{GF}(q)$. The $\mathrm{CU}$ takes

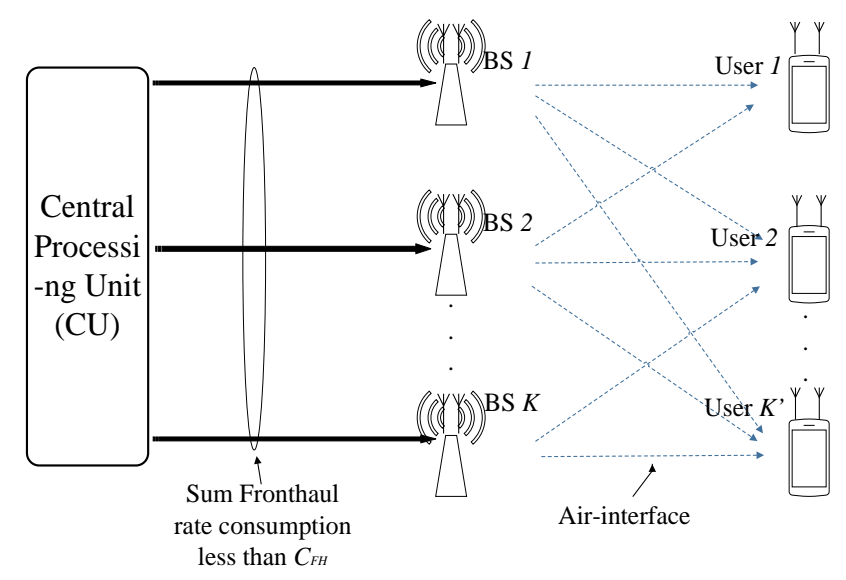

Fig. 1. Block diagram of an $K$-base station $K^{\prime}$-user downlink DMIMO system with rate-constrained front-haul.

in the $K^{\prime}$ message sequences and produces $K$ functions of them, generally written as

$$
\mathbf{u}_{i}=\psi_{i}\left(\mathbf{b}_{1}, \cdots, \mathbf{b}_{K^{\prime}}\right), i=1,2, \cdots, K .
$$

The signal $\mathbf{u}_{i}=\psi_{i}\left(\mathbf{b}_{1}, \cdots, \mathbf{b}_{K^{\prime}}\right)$ is forwarded to the $i$ th BS through the $i$ th FH link. The FH consumption is given by $H\left(\mathbf{u}_{i}\right)$ where $H(\cdot)$ denotes the entropy function.

Denote by $C_{F H}$ the maximum rate that can be supported by the $K \mathrm{FH}$ links in total for one block of transmission [11], [26] [14]. The rate-constraint under consideration is given by

$$
\sum_{i=1}^{K} H\left(\mathbf{u}_{i}\right) \leq C_{F H} .
$$

Error free transmission of the signal (1) over the FH links is assumed if the rate constraint (2) is met. In this paper, we only consider a total FH rate constraint as in [4]. Our proposed scheme and methods apply to the scenario with individual front-haul rate constraints.

\section{B. Air-Interface Layer}

Upon obtaining $\mathbf{u}_{i}$, BS $i$ 's encoder maps $\mathbf{u}_{i}$ into a size $N_{i}$ by $n$ space-time signal matrix $\mathbf{X}_{i}$. Denote the encoding functions by

$$
\mathbf{X}_{i}=\varepsilon_{i}\left(\mathbf{u}_{i}\right), i=1,2, \cdots, K .
$$

A total power constraint is given by ${ }^{1}$

$$
\sum_{i=1}^{K} E\left(\left|\mathbf{x}_{i}^{T}[t] \mathbf{x}_{i}[t]\right|^{2}\right) \leq E_{\text {total }}
$$

where $\mathbf{x}_{i}[t]$ denotes the $t$ th column of $\mathbf{X}_{i}$, representing the signal vector transmitted by BS $i$ 's multiple antennas at time instant $t, t=1, \cdots, n$.

\footnotetext{
${ }^{1}$ We only focus on the case with total power constraint. Our scheme can be extended to individual power constraint.
} 
The signal received by the $j$ th user in a block of $n$ channeluse is

$$
\mathbf{Y}_{j}=\sum_{i=1}^{K} \mathbf{H}_{j, i} \mathbf{X}_{i}+\mathbf{Z}_{j}, j=1,2, \cdots, K^{\prime},
$$

where $\mathbf{H}_{j, i}$ denotes the channel coefficient matrix from BS $i$ to User $j$, and $\mathbf{Z}_{j}$ denotes the additive white Gaussian noise (AWGN) with i.i.d. entries of zero mean and variance $\frac{N_{0}}{2}$ per real-dimension. The per-node signal-to-noise ratio (SNR) is defined as $\rho \triangleq \frac{E_{\text {total }}}{N_{0} K}$.

In this paper, a block fading channel is considered, i.e. the channel coefficients remain constant over a block of $n$ symbols and vary over blocks. Global channel state information (CSI) and symbol synchronization is assumed.

User $j$ decodes its desired message based on $\mathbf{Y}_{j}$. The decoding process is written as

$$
\widehat{\mathbf{b}}_{j}=\operatorname{dec}_{j}\left(\mathbf{Y}_{j}\right) \text {. }
$$

where $\widehat{\mathbf{b}}_{j}$ denotes the decision. An error is declared if $\widehat{\mathbf{b}}_{j} \neq$ $\mathbf{b}_{j}$ for any $j \in\left\{1, \cdots, K^{\prime}\right\}$.

\section{Performance Metric}

Consider given channel coefficient matrices $\mathbf{H}_{j, i}, i=$ $1, \cdots, K, j=1, \cdots, K^{\prime}$, and given $\mathrm{FH}$ rate-constraints $C_{F H}$. The information rates of the users' messages are denoted by $R_{j}=\frac{m_{j}}{n} \log _{2} q, j=1, \cdots, K^{\prime}$. A $\left(R_{1}, \cdots, R_{K^{\prime}}, n, P_{e}\right)$ code consists of the following:

1. $K \mathrm{FH}$ functions $\psi_{1}(\cdot), \cdots, \psi_{K}(\cdot)$ at the CU that satisfy the $\mathrm{FH}$ rate-constraint(2).

2. $K$ encoding functions $\varepsilon_{1}(\cdot), \cdots, \varepsilon_{K}(\cdot)$ at the $K$ BSs as specified in (3).

3. $K^{\prime}$ decoding functions $\operatorname{dec}_{1}(\cdot), \cdots, \operatorname{dec}_{K^{\prime}}(\cdot)$ at the $K^{\prime}$ users as specified in (6).

4. An error probability $P_{e}$, given by

$$
P_{e}=\operatorname{Pr}\left\{\left[\widehat{\mathbf{b}}_{1}, \cdots, \widehat{\mathbf{b}}_{K^{\prime}}\right] \neq\left[\mathbf{b}_{1}, \cdots, \mathbf{b}_{K^{\prime}}\right]\right\} .
$$

A information rate tuple $\left(R_{1}, \cdots, R_{K^{\prime}}\right)$ is said to be achievable if, for any $\epsilon>0$, there exists a $\left(R_{1}, \cdots, R_{K^{\prime}}\right.$, $n, P_{e}$ ) code such that $P_{e} \leq \epsilon$ as the block length $n \rightarrow \infty$.

A degrees of freedom (DoF) tuple $\left(d_{1}, \cdots, d_{K^{\prime}}\right)$ is said to be achievable if the rate scales like

$$
\lim _{\rho \rightarrow \infty} \frac{R_{j}}{\log \rho}=d_{j}
$$

for $j=1, \cdots, K^{\prime}$. An achievable total DoF is given by $d=$ $\sum_{j=1}^{K^{\prime}} d_{j}$

\section{Problem at a Glance}

This paper primarily focus on that the $\mathrm{FH}$ rate-constraint and information capacity of the air-interface are comparable, i.e., they are of the same order of magnitude. In addition, this paper focus on constant (or block fading) channel with no symbol extension ${ }^{2}$.

\footnotetext{
${ }^{2}$ Our proposed can be extended to symbol-extension for time-varying channel. Note that alignment in spatial dimension through multi-antenna is more robust to practical limitations such as frequency offsets than alignment in time or frequency [27].
}

Consider the symmetric setup with $K=K^{\prime}$ and $N=N^{\prime}$. First, if CU forwards the following

$$
\mathbf{u}_{i}=\mathbf{b}_{i}, i=1,2, \cdots, K
$$

to the $\mathrm{BSs}$, the D-MIMO reduces to a $K$-user interference channel. The total FH consumption is $\sum_{i=1}^{K} H\left(\mathbf{b}_{i}\right)$. It is wellknown that interference alignment (IA) with infinite symbolextension is necessary to achieve the DoF of such an interference channel. The total DoF is $\frac{N}{2} K$ for infinite symbolextension, and could be far less than $\frac{N}{2} K$ for small symbolextension in practice [28].

Second, note that if $\mathrm{CU}$ forwards the following functions

$$
\mathbf{u}_{i}=\left(\mathbf{b}_{1}, \cdots, \mathbf{b}_{K}\right), i=1,2, \cdots, K
$$

to the BSs, the D-MIMO scheme reduces to MIMO broadcast channel with $\sum_{i=1}^{K} N_{i}$ co-located transmit antennas. The total FH consumption is $\sum_{i=1}^{K} H\left(\mathbf{b}_{1}, \cdots, \mathbf{b}_{K}\right)$. The DoF is given by $N K$.

Comparing the above two cases, we see that if the FH capacity constraint increases from $\sum_{i=1}^{K} H\left(\mathbf{b}_{i}\right)$ to $K \sum_{i=1}^{K} H\left(\mathbf{b}_{i}\right)$, the DoF increases from a value less than $\frac{N}{2} K$ to exactly $N K$. This gives rise to the following set of questions: what is the DoF of DMIMO if the FH capacity is somewhere between $\sum_{i=1}^{K} H\left(\mathbf{b}_{i}\right)$ and $K \sum_{i=1}^{K} H\left(\mathbf{b}_{i}\right)$ ? Is an IA-type of approach necessary to achieve the DoF of DMIMO in that scenario? This paper attempts to answer these questions via studying a new network coding based D-MIMO broadcasting scheme presented in the subsequent section.

\section{Proposed Distributed MiMO BroAdCAsting SCHEME}

The proposed scheme is depicted in Fig. 2.

\section{A. Pre Network Coding at Central Unit}

1) Data Streams: The message sequence $\mathbf{b}_{j}$ for User $j$ is truncated into $L_{j}^{\prime}$ segments of row vectors $\mathbf{b}_{j, 1}, \cdots, \mathbf{b}_{j, L_{i}^{\prime}}$, for spatial multiplexing purpose. The lengths of the truncated message sequences are respectively denoted by $m_{j, 1}, \cdots, m_{j, L_{j}^{\prime}}$, with $\sum_{l^{\prime}=1}^{L_{j}^{\prime}} m_{j, l^{\prime}}=m_{j}$. Each of $\mathbf{b}_{j, 1}, \cdots, \mathbf{b}_{j, L_{i}^{\prime}}$ is referred to as a message stream. The rate associated with a message stream $\mathbf{b}_{j, l^{\prime}}$ is

$$
R_{j, l^{\prime}}=\frac{m_{j, l^{\prime}}}{n} \log _{2} q .
$$

The message streams for User $j$ are collectively represented by

$$
\mathbf{B}_{j}=\left[\overline{\mathbf{b}}_{j, 1}^{T}, \cdots, \overline{\mathbf{b}}_{j, L_{j}}^{T}\right]^{T}, j=1, \cdots, K^{\prime},
$$




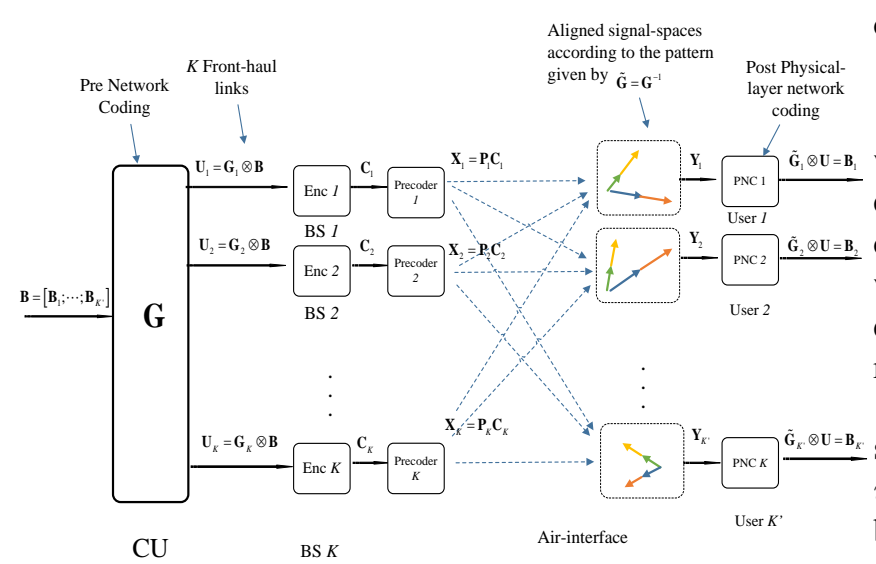

Fig. 2. Block diagram of the proposed D-MIMO broadcasting with signalspace alignment and reverse compute-and-forward.

where $\overline{\mathbf{b}}_{j, l^{\prime}}$ is formed by padding $\mathbf{b}_{j, l^{\prime}}$ with $m_{\max }-m_{j, l^{\prime}}$ zeros, where $m_{\max } \triangleq \max _{j, l^{\prime}} m_{j, l^{\prime}}$. Denote

$$
\mathbf{B}=\left[\mathbf{B}_{1}^{T}, \cdots, \mathbf{B}_{K^{\prime}}^{T}\right]^{T}
$$

All $L^{\prime}=\sum_{j=1}^{K^{\prime}} L_{j}^{\prime}$ message streams for all $K^{\prime}$ users are now collected in the $L^{\prime}$ rows of $\mathbf{B}$.

2) A Single Network-Coded (NC) Message Sequence: A finite field linear combination of the users' message sequences, referred to as a NC message sequence, is written as

$$
\mathbf{u}=\mathbf{g} \otimes \mathbf{B}
$$

where " $\otimes$ " denotes the multiplication operation in $\operatorname{GF}(q)$. Here, the row vector $\mathrm{g}$ is referred to as a NC coefficient vector, whose $L^{\prime}$ entries specify the coefficients of the linear combination.

a) User Index Function: For the ease of illustration, define the following User index function

$$
J\left(j, l^{\prime}\right) \triangleq \sum_{\gamma=1}^{j-1} L_{j}^{\prime}+l^{\prime}
$$

The $l^{\prime}$ th message stream of User $j$, also called the $\left(j, l^{\prime}\right)$ th message stream, is now located at the $J\left(j, l^{\prime}\right)$-th row of B. The NC coefficient corresponding to the $\left(j, l^{\prime}\right)$ th message stream is specified by the $J\left(j, l^{\prime}\right)$-th entry of $\mathbf{g}$.

Let $\tau=J\left(j, l^{\prime}\right)$. The inverse of the User index function is defined as

$$
J^{-1}(\tau) \triangleq j \text { if } \sum_{\gamma=1}^{j-1} L_{\gamma}^{\prime}<\tau \leq \sum_{\gamma=1}^{j} L_{\gamma}^{\prime}
$$

The $\tau$ th row of $\mathbf{B}$ belongs to User $J^{-1}(\tau)$. The $\tau$ th entry of $\mathrm{g}$ is w.r.t. User $J^{-1}(\tau)$. b) NC Message Rate: The NC message rate per channel-use w.r.t. $\mathbf{u}$ is defined as

$$
\widetilde{R} \triangleq \max _{j, l^{\prime}: g\left[J\left(j, l^{\prime}\right)\right] \neq 0} \frac{m_{j, l^{\prime}}}{n} \log _{2} q
$$

where $g\left[J\left(j, l^{\prime}\right)\right]$ denotes the $J\left(j, l^{\prime}\right)$-th entry of $\mathrm{g}$. To explain (17), note that the message streams with zero $\mathrm{NC}$ coefficients are irrelevant in the NC message $u$. In other words, only those message streams with non-zero NC coefficients are taken into account, and the one with maximum rate determines the $\mathrm{NC}$ message rate (17).

3) Multiple NC Message Sequences: In the proposed scheme, the CU forwards $L_{i} \mathrm{NC}$ message sequences to BS $i$. These NC message sequences are collectively represented by

$$
\mathbf{U}_{i}=\left[\begin{array}{c}
\mathbf{u}_{i, 1} \\
\vdots \\
\mathbf{u}_{i, L_{i}}
\end{array}\right]=\left[\begin{array}{c}
\mathbf{g}_{i, 1} \otimes \mathbf{B} \\
\vdots \\
\mathbf{g}_{i, L_{i}} \otimes \mathbf{B}
\end{array}\right], i=1, \cdots, K,
$$

where $\mathbf{g}_{i, l}$ denotes the coefficient vector of the $l$ th $\mathrm{NC}$ message sequence forwarded to BS $i, l=1, \cdots, L_{i}$.

Similar to (17), the NC message rate of $\mathbf{u}_{i, l}$ is given by

$$
\widetilde{R}_{i, l} \triangleq \max _{j, l^{\prime}: g_{i, l}\left[J\left(j, l^{\prime}\right)\right] \neq 0} \frac{m_{j, l^{\prime}}}{n} \log _{2} q .
$$

The NC messages are delivered to the $K$ BSs error-free if the $\mathrm{FH}$ rate constraint

$$
\sum_{i=1}^{K} \sum_{l=1}^{L_{i}} \widetilde{R}_{i, l}<\bar{C}_{F H}
$$

is satisfied, where $\bar{C}_{F H} \triangleq \frac{C_{F H}}{n}$ denotes the normalized FH rate-constraint per channel-use.

Without loss of generality, we assume $L_{1} \geq L_{2} \geq \cdots \geq$ $L_{K}$.The total number of NC message sequences forwarded to all BSs is $L=\sum_{i=1}^{K} L_{i}$.

4) NC Generator Matrix: Let

$$
\mathbf{G}_{i}=\left[\mathbf{g}_{i, 1}^{T}, \cdots, \mathbf{g}_{i, L_{i}}^{T}\right]^{T}, i=1, \cdots, K
$$

whose rows collect coefficient vectors of the $L_{i}$ NC message sequences forwarded to $\mathrm{BS} i$. Then Eq. (18) is written as

$$
\mathbf{U}_{i}=\mathbf{G}_{i} \otimes \mathbf{B} .
$$

Further, let

$$
\mathbf{G}=\left[\mathbf{G}_{1}^{T}, \cdots, \mathbf{G}_{K}^{T}\right]^{T}
$$

which collects all $L \mathrm{NC}$ coefficient vectors w.r.t. all BSs. The $L \mathrm{NC}$ message sequences forwarded to all BSs can now be concisely written as

$$
\mathbf{U}=\left[\mathbf{U}_{1}^{T}, \cdots, \mathbf{U}_{K}^{T}\right]^{T}=\mathbf{G} \otimes \mathbf{B} .
$$

We refer to $\mathbf{G}$ as a $N C$ generator matrix. The size of $\mathbf{G}$ is $L$ by $L^{\prime}$.

In this paper, we consider that $\mathbf{G}$ is a square matrix of full rank, i.e., $L=L^{\prime}$ and $\operatorname{Rank}(\mathbf{G})=L$ in $\operatorname{GF}(q)$. For $L<L^{\prime}$, it is impossible to reconstruct $\mathbf{B}$ from $\mathbf{U}$. For $L>L^{\prime}$, there is at least one NC message that is linearly dependent on other NC messages. This may lead to a waste in the $\mathrm{FH}$ consumption. Our approach can be extended to the case where $L>L^{\prime}$. 


\section{B. Encoding at the Base Stations}

BS $i$ carries out $L_{i}$ independent channel-code encoding and modulation operations over its $\mathrm{NC}$ message sequences $\mathbf{u}_{i, 1}, \cdots, \mathbf{u}_{i, L_{i}}$, yielding length- $n$ coded-modulated sequences $\mathbf{c}_{i, 1}, \cdots, \mathbf{c}_{i, L_{i}}$ respectively. The overall rate of the channel coding and modulation for $\mathbf{u}_{i, l}$ is determined by the NC message rate $\widetilde{R}_{i, l}$. Note that each coding-modulation is over a NC message sequence. This is different from a conventional system where the coding-modulation is over a data message sequence.

We refer to $\mathbf{c}_{i, l}$ as a spatial NC stream ${ }^{3}$. Each of the spatial NC streams $\mathbf{c}_{i, 1}, \cdots, \mathbf{c}_{i, L_{i}}$ of BS $i$ carries one of the NC messages sequences $\mathbf{u}_{i, 1}, \cdots, \mathbf{u}_{i, L_{i}}$, respectively.

The underlying channel coding and modulation must support PNC (or CF). From a theoretical aspect, there exists Voronoi-region nested lattice codes, which are simultaneously "Poltyrev-good" and "Roger-good", that can be used to prove some recently established achievable rate of PNC or CF [18] [15]. In practice, non-binary irregular repeat modulationcoded PNC scheme with iterative believe propagation decoding algorithm can be employed, which has been shown to perform very close to the theoretical rate limit [29].

The coded-modulated sequences are collectively written as

$$
\mathbf{C}_{i}=\left[\mathbf{c}_{i, 1}^{T}, \cdots, \mathbf{c}_{i, L_{i}}^{T}\right]^{T}, i=1, \cdots, K .
$$

After the channel coding and modulation, BS $i$ performs a linear precoding, yielding

$$
\mathbf{X}_{i}=\mathbf{P}_{i} \mathbf{C}_{i}, i=1, \cdots, K .
$$

The precoding matrices of all BSs are collected by $\mathbf{P}=\left[\mathbf{P}_{1}, \cdots, \mathbf{P}_{K}\right]$. The signal-spaces of the spatial NC streams are determined by the columns of $\mathbf{P}$. Let the entries of $\mathbf{C}_{i}$ be normalized. Then, the power constraint in (4) becomes

$$
\sum_{i=1}^{K} \operatorname{Tr}\left(\mathbf{P}_{i} \mathbf{P}_{i}^{H}\right) \leq E_{\text {total }}
$$

The $K$ BSs transmit $\mathbf{X}_{1}, \cdots, \mathbf{X}_{K}$ simultaneously. User $j$ receives $\mathbf{Y}_{j}$, as specified in (5).

We next present the design of $\mathbf{P}$. The following notations will be used.

a) For the ease of illustration, a $B S$ index function is defined as

$$
I(i, l) \triangleq \sum_{\gamma=1}^{i-1} L_{i}+l .
$$

The $l$ th spatial NC stream of BS $i$, refer to the $(i, l)$ th spatial NC stream, is now located at the $I(i, l)$ th row of $\mathbf{U}$.

Let $\tau=I(i, l)$. The inverse of the BS index function is defined as

$$
I^{-1}(\tau) \triangleq i \text { if } \sum_{\gamma=1}^{i-1} L_{\gamma}<\tau \leq \sum_{\gamma=1}^{i} L_{\gamma}
$$

The $\tau$ th row of $\mathbf{U}$ now belongs to BS $I^{-1}(\tau)$.

\footnotetext{
${ }^{3}$ This name is used to distinguish it from a message stream at the $\mathrm{CU}$.
}

b) The columns of $\mathbf{P}_{i}$ are denoted by $\mathbf{p}_{i, l}, l=1, \cdots, L_{i}$. The columns of $\mathbf{P}=\left[\mathbf{P}_{1}, \ldots, \mathbf{P}_{K}\right]$ are denoted by $\mathbf{p}_{\tau}, \tau=$ $1, \cdots, L$, where $\mathbf{p}_{i, l}=\mathbf{p}_{I(i, l)}$ from the definition of BS index-function (28).

c) Let

$$
\widetilde{\mathbf{G}}=\mathbf{G}^{-1}
$$

where the matrix inverse operation takes place in $\operatorname{GF}(q)$. We refer to $\widetilde{\mathbf{G}}$ as the inverse NC generator matrix. The blocks of $\widetilde{\mathbf{G}}$ are denoted by

$$
\widetilde{\mathbf{G}}=\left[\widetilde{\mathbf{G}}_{1}^{T}, \cdots, \widetilde{\mathbf{G}}_{K^{\prime}}^{T}\right]^{T},
$$

where

$$
\widetilde{\mathbf{G}}_{j}=\left[\widetilde{\mathbf{g}}_{j, 1}^{T}, \cdots, \widetilde{\mathbf{g}}_{j, L_{j}^{\prime}}^{T}\right]^{T} .
$$

In our proposed scheme, $\widetilde{\mathbf{g}}_{j, 1}, \cdots, \widetilde{\mathbf{g}}_{j, L_{j}^{\prime}}$ will be used as the coefficient vectors in computing the linear combinations of the NC messages at User $j$. We emphasize that $\widetilde{\mathbf{g}}_{j, l^{\prime}}$ is for the post PNC operation at a user node, while $\mathbf{g}_{i, l}$ is for the pre network coding operation at the CU.

1) Signal-space Alignment Precoding: The signal-spaces of two vectors $\mathbf{a}$ and $\mathbf{b}$ are said to be aligned if $\mathbf{b}=c \cdot \mathbf{a}$, where $c$ is a non-zero constant scalar. Let $\mathbf{a} \| \mathbf{b}$ represents that $\mathbf{b}=c \cdot \mathbf{a}$.

For $\widetilde{\mathbf{g}}_{j, l^{\prime}}$, define a support set

$$
\mathcal{T}\left(\widetilde{\mathbf{g}}_{j, l^{\prime}}\right) \triangleq\left\{\tau \in\left\{1, \cdots, L^{\prime}\right\}: \widetilde{g}_{j, l^{\prime}}[\tau] \neq 0\right\}
$$

where $\widetilde{g}_{j, l^{\prime}}[\tau]$ denotes the $\tau$ th entry of $\widetilde{\mathbf{g}}_{j, l^{\prime}}$. This support set indicates the spatial NC streams that are relevant in the signal-space alignment depicted below.

Definition 1: A set of spatial NC streams is said to form a bin if their signal-spaces are aligned at a specific user node.

In our proposed scheme, we create $L_{j}^{\prime}$ bins for User $j$. Specifically, the "binning" is given by

$$
\mathcal{B}_{j, l^{\prime}}=\left\{\mathbf{c}_{i, l} \forall i, l: I(i, l) \in \mathcal{T}\left(\widetilde{\mathbf{g}}_{j, l^{\prime}}\right)\right\}, l^{\prime}=1, \cdots, L_{j}^{\prime} .
$$

The $l^{\prime}$ th bin at User $j$ contains the spatial NC streams w.r.t. the non-zero elements in $\widetilde{\mathbf{g}}_{j, l^{\prime}}$.

By definition, the signal-spaces of the spatial NC streams in the same bin are aligned at User $j$. This leads to the following signal-space alignment condition:

$\mathbf{H}_{j, I^{-1}(\tau)} \mathbf{p}_{\tau} \| \mathbf{H}_{j, I^{-1}\left(\tau^{\prime}\right)} \mathbf{p}_{\tau^{\prime}} \forall\left(\tau, \tau^{\prime}\right) \in \mathcal{T}\left(\widetilde{\mathbf{g}}_{j, l^{\prime}}\right), j=1, \cdots, K^{\prime}$.

Note that the binning, or the pattern of signal-space alignment, is determined by inverse NC generator matrix $\widetilde{\mathbf{G}}$.

Remark 1: There are up to $N_{j}^{\prime}$ bins at User $j$, which stores $L=\sum_{i=1}^{K} L_{i}>N_{j}^{\prime}$ spatial NC streams. Bin $l^{\prime}$ contains only those spatial NC streams w.r.t. non-zero entries in $\widetilde{\mathbf{g}}_{j, l^{\prime}}$. The cardinality of each bin is equal to the number of non-zero entries of the corresponding coefficient vector, i.e., $\left|\mathcal{B}_{j, l^{\prime}}\right|=$ $\left|\mathcal{T}\left(\widetilde{\mathbf{g}}_{j, l^{\prime}}\right)\right|$. In addition, at each user, a spatial NC stream cannot appear in two different bins, i.e., $\mathcal{B}_{j, l^{\prime}} \cap \mathcal{B}_{j, \bar{l}^{\prime}}=\emptyset$ for $\bar{l}^{\prime} \neq l^{\prime}$. Moreover, all NC messages are contained in the $L_{j}$ bins, without missing any. These impose certain structural properties on the NC generator matrix, as we shall present in Section IV. B. 
2) Scaled and Equal-gain Signal-space Alignment: A $s$ caled signal-space alignment satisfies

$$
\begin{aligned}
& \mathbf{H}_{j, I^{-1}(\tau)} \mathbf{p}_{\tau} \| \mathbf{H}_{j, I^{-1}\left(\tau^{\prime}\right)} \mathbf{p}_{\tau^{\prime}}, \\
& \frac{\left\|\mathbf{H}_{j, I^{-1}(\tau)} \mathbf{p}_{\tau}\right\|}{\left\|\mathbf{H}_{j, I^{-1}\left(\tau^{\prime}\right)} \mathbf{p}_{\tau^{\prime}}\right\|}=\frac{\widetilde{g}_{j, l^{\prime}}[\tau]}{\widetilde{g}_{j, l^{\prime}}\left[\tau^{\prime}\right]}, \forall\left(\tau, \tau^{\prime}\right) \in \mathcal{T}\left(\widetilde{\mathbf{g}}_{j, l^{\prime}}\right) .
\end{aligned}
$$

With it, the quantization error due to the non-integer ratio penalty in CF is removed [15].

In particular, if the entries of $\widetilde{\mathbf{G}}$ belong to $\{0,1\}$, (36) becomes

$$
\mathbf{H}_{j, I^{-1}(\tau)} \mathbf{p}_{\tau}=\mathbf{H}_{j, I^{-1}\left(\tau^{\prime}\right)} \mathbf{p}_{\tau^{\prime}} \forall\left(\tau, \tau^{\prime}\right) \in \mathcal{T}\left(\widetilde{\mathbf{g}}_{j, l^{\prime}}\right) .
$$

This is called equal gain signal-space alignment, which will be discussed in Section IV. D.

\section{User Nodes' Decoding}

1) Receiver Shaping: At a user, the bins are not necessarily orthogonal to each other. To decouple these bins, User $j$ first carries out a linear transformation over $\mathbf{Y}_{j}$ given by

$$
\mathbf{Q}_{j}=\mathbf{F}_{j}^{H} \mathbf{Y}_{j}, j=1, \cdots, K^{\prime} .
$$

Denote by $\mathbf{f}_{j, l^{\prime}}$ the $l^{\prime}$-th column of $\mathbf{F}_{j}$. The vector $\mathbf{f}_{j, l^{\prime}}^{H}$ projects $\mathbf{Y}_{j}$ to the subspace which is perpendicular to the directions of other bins (which are irrelevant to the computation of the $l^{\prime}$ th linear combination of the NC messages). Specifically, let $t\left(j, l^{\prime}\right)$ be the first element of $\mathcal{T}\left(\widetilde{\mathbf{g}}_{j, l^{\prime}}\right)$. Then, the filter $\mathbf{f}_{j, l^{\prime}}$ satisfies

$\mathbf{f}_{j, l^{\prime}}^{H} \mathbf{H}_{j, \eta} \mathbf{p}_{1, \eta}=0 \forall \eta \in\left\{1, \cdots, L_{1}^{\prime}\right\}, \eta \neq t\left(j, l^{\prime}\right),\left\|\mathbf{f}_{j, l^{\prime}}\right\|=1$.

Let the singular value decomposition of the matrix

$\boldsymbol{\Omega}_{j, l^{\prime}}=\left[\mathbf{H}_{j, 1} \mathbf{p}_{1,1}, \ldots, \mathbf{H}_{j, t\left(j, l^{\prime}\right)-1} \mathbf{p}_{1, t\left(j, l^{\prime}\right)-1}, \mathbf{H}_{j, t\left(j, l^{\prime}\right)+1} \mathbf{p}_{1, t(j,}\right.$

be given by

$$
\boldsymbol{\Omega}_{j, l^{\prime}}=\mathbf{U}_{j, l^{\prime}}^{\Omega} \boldsymbol{\Sigma}_{j, l^{\prime}}\left(\mathbf{V}_{j, l^{\prime}}^{\Omega}\right)^{H}
$$

where $\boldsymbol{\Sigma}_{j, l^{\prime}}$ is the diagonal matrix consisting of the singular values. It can be shown that the rank of $\boldsymbol{\Omega}_{j, l^{\prime}}$ is $L_{j}^{\prime}-1$ with probability 1 . The filter $\mathbf{f}_{j, l^{\prime}}$ is any linear combination of the columns of $\mathbf{U}_{j, l^{\prime}}^{\Omega}$ that corresponds to the zero singular values.

2) Decoding with Post Physical-layer Network Coding: Denote by $\mathbf{q}_{j, l^{\prime}}$ the $l^{\prime}$-th row of $\mathbf{Q}_{j}$. After the receiver shaping, the bins are decoupled and the $L_{j}^{\prime}$ signal sequences $\mathbf{q}_{j, 1}, \cdots, \mathbf{q}_{j, L_{j}^{\prime}}$ are non-interfering where each involves a number of aligned spatial NC streams. Based on that, User $j$ employs $L_{j}^{\prime}$ independent PNC operations to compute $L_{j}^{\prime}$ linear combinations over the NC message sequences respectively. Specifically, the associated coefficient vectors are $\widetilde{\mathbf{g}}_{j, 1}, \cdots, \widetilde{\mathbf{g}}_{j, L_{j}^{\prime}}$ taken from $\widetilde{\mathbf{G}}$, and the PNC operation aims to compute

$$
\mathbf{v}_{j, l^{\prime}}=\widetilde{\mathbf{g}}_{j, l^{\prime}} \otimes \mathbf{U}, l^{\prime}=1, \cdots, L_{j}^{\prime} .
$$

We note that $\mathbf{v}_{j, l^{\prime}}=\mathbf{b}_{j, l^{\prime}}$ for all $l^{\prime}$ and $j$. That is, the linear combination of the NC message sequences is identical to the desired message of that user. The outputs of the $L_{j}^{\prime}$ PNC operations at $\mathrm{BS} j$ is

$$
\widehat{\mathbf{v}}_{j, l^{\prime}}=\widehat{\mathbf{b}}_{j, l^{\prime}}, l^{\prime}=1, \cdots, L_{j}^{\prime} .
$$

The above follows from that the post PNC operation exactly equalizes (or reverses) the pre network coding operation done by the $\mathrm{CU}$, owing to $\widetilde{\mathbf{G}}=\mathbf{G}^{-1}$. To see this, denote

$$
\mathbf{V}_{j}=\left[\begin{array}{c}
\mathbf{v}_{j, 1} \\
\vdots \\
\mathbf{v}_{j, L_{j}}
\end{array}\right]=\left[\begin{array}{c}
\widetilde{\mathbf{g}}_{j, 1} \otimes \mathbf{U} \\
\vdots \\
\widetilde{\mathbf{g}}_{j, L_{j}} \otimes \mathbf{U}
\end{array}\right] .
$$

Further, let $\mathbf{V}=\left[\mathbf{V}_{1}^{T}, \cdots, \mathbf{V}_{K}^{T}\right]^{T}$. Then

$$
\mathbf{V}=\widetilde{\mathbf{G}} \otimes \mathbf{U}=\widetilde{\mathbf{G}} \otimes \mathbf{G} \otimes \mathbf{B}=\mathbf{B}
$$

\section{Comments on the Proposed Scheme and Difference to Existing Schemes}

What motivate the proposed scheme are: a) there lacks sufficient receive signal-spaces for accommodating the aggressively large amount of spatial streams in D-MIMO and b) there is a potential coding gain or even DoF gain that can be achieved by PNC or CF [30]. First, note that there are much more spatial streams than the dimension of the receive signalspace at each user nodes. For the uplink scenario, one way to address this is compress the dimension of the signal streams by deliberately align their signal spaces. Second, for each bin of aligned signal-space, a superposition of multiple streams is observed. PNC (or CF) is used to directly reconstruct the linear message combinations. Then, each spatial stream can deliver one full DoF, which leads to significantly greater DoF than complete decoding all individual streams [18], [15]. The CU can solve those linear message combinations and eventually recover all users' messages. More details about

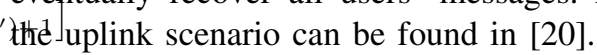

Yet, in the downlink scenario considered in this paper, the key challenge is that the user nodes, where PNC or CF is conducted, are already the final destinations. Thus, the computed linear message combinations at each user node have to be identical to the desired messages. To address this challenge, we borrow the notion of reverse computeand-forward [25]. In our proposed scheme, the CU preconvert the messages sequence $\mathbf{B}$ into network coded version $\mathbf{U}=\mathbf{G} \otimes \mathbf{B}$. At the user nodes, the post PNC operation computes $\mathbf{G}^{-1} \otimes \mathbf{U}=\mathbf{B}$ in a distributed manner. This compensates (or equalizes) the pre network coding done at the $\mathrm{CU}$, and the computed linear message combinations are indeed identical to the desired messages. By binning the spatial NC streams, our proposed signal-space alinement precoding essentially provides a bridge between the pre network coding and the post PNC. We note that such a bridging is valid only if the NC generator matrix satisfies certain structural properties, which will be studies in the next section.

We note that our proposed approach differs from the integer-forcing IA scheme [21] and our previous work [20] in the following aspects. The integer-forcing IA scheme was developed for the interference channel only. The notion is to 
decode the integer combinations of the interferences in the "waste bin", which is then successively canceled to make the desired signal "cleaner". Our proposed scheme differs from the channel integer alignment scheme in [31] in the following aspects: First, [31] considered two-user cognitive interference network and a two-user Gaussian interference channel, which are different system models. Note that our proposed scheme applies to a general $K$-user system, while that in [31] only applies to a two-user system. Second, at the heart of our proposed scheme lies a pre-network coding generator matrix, whose inverse determines the pattern of signal-space alignment and reverse CF. The existence of the pre network coding generator matrix is a pre-requisite for implementing signal space alignment in the D-MIMO system.

\section{Achievable Rate Analysis}

In this section, we analyze the achievable information rate of the proposed scheme. Throughout this section, we assume that $N_{1}=\cdots=N_{K}=N$ and $N_{1}^{\prime}=N_{2}^{\prime}$ $=\cdots=N_{K^{\prime}}^{\prime}=N^{\prime 4}$. We consider that the precoding matrices derived in the previous section are properly scaled to satisfy the power constraint (27), which is relaxed in the sequel.

\section{A. Achievable Rate Conditioned on Aligned Signal-spaces}

1) Achievable NC Message Rate: Recall that the $L \mathrm{NC}$ message sequences and the $L^{\prime}$ message sequences are related by the one-to-one mapping

$$
\mathbf{U}=\mathbf{G} \otimes \mathbf{B} .
$$

The NC message rate w.r.t. $\mathbf{g}_{i, l}$ (the $I(i, l)$ th row of $\mathbf{G}$ ) has been defined as in (19).

Definition 2: A NC message rate-tuple is said to be achievable if there exists encoding functions over the NC messages at the $K$ BSs and decoding functions at the $K^{\prime}$ users such that the error probability $P_{e}=$ $\operatorname{Pr}\left\{\left[\widehat{\mathbf{b}}_{1}, \cdots, \widehat{\mathbf{b}}_{K^{\prime}}\right] \neq\left[\mathbf{b}_{1}, \cdots, \mathbf{b}_{K^{\prime}}\right]\right\} \leq \epsilon$, as $n \rightarrow \infty$.

$$
\eta_{i, l}^{j} \triangleq l^{\prime}: g_{j, l^{\prime}}[I(i, l)] \neq 0,
$$

which locates the $l^{\prime}$ th bin (at User $j$ ) that contains the $(i, l)$ th spatial NC stream.

Proposition 1: The following $L$-tuple of NC message rates is achievable

$$
\begin{aligned}
\widetilde{R}_{i, l} & <\min _{j \in\left\{1, \cdots, K^{\prime}\right\}} \log _{2}\left(\left|\mathbf{f}_{j, \eta_{i, l}^{j}} \mathbf{H}_{j, i} \mathbf{p}_{i, l}\right|^{2} \rho\right)^{+} \log _{2} q, \\
& l \in\left\{1, \cdots, L_{i}\right\}, i \in\{1, \cdots, K\}
\end{aligned}
$$

given that $\mathbf{p}_{i, l}, l=1, \cdots, L_{i}, i=1, \cdots, K$, satisfy the signal-space alignment condition in (36).

Proof. Without loss of generality, let the $L$ coded-modulated streams $\mathbf{c}_{i, l}, l=1, \ldots, L_{i}, i=1, \ldots, K$, be arranged in a descending order according their rates. The coding and modulation is carried out using a nested lattice code for each stream, described as follows. Consider a chain of lattices

\footnotetext{
${ }^{4}$ Our treatment can be straightforwardly extended to the case where the wireless terminals have different numbers of antennas.
}

$\Lambda_{S} \subseteq \Lambda_{L} \subseteq \cdots \subseteq \Lambda_{1}$. A nested lattice code is a lattice code which uses as codewords and the Voronoi region of the coarse $\Lambda_{S}$ as a shaping region. The $L$ fine lattices $\Lambda_{1}, \ldots, \Lambda_{L}$ are used for channel coding for the $L$ data streams, respectively. For the nesting of $\Lambda_{l} / \Lambda_{S}, l=1, \cdots, L$, the set of codewords is given by $\left\{\Lambda_{l} \bmod \Lambda_{S}\right\}=\left\{\Lambda_{l} \cap \mathcal{V}_{S}\right\}$ where $\mathcal{V}_{S}$ denotes the fundamental Voronoi region of the coarse lattice $\Lambda_{S}$. A dithering operation is performed to gurantee that the signal is uniformly distributed in $\mathcal{V}_{S}$. Note that in our proposed scheme the coarse lattice $\Lambda_{S}$ is common to all $L$ data streams.

Recall the assumption that there exists $\widetilde{\mathbf{G}}$ and $\mathbf{p}_{i, l}, l=$ $1, \cdots, L_{i}, i=1, \cdots, K$, such that the signal-space alignment is guaranteed. Consider the $l^{\prime}$ th bin at User $j$ that contains the $l$ th spatial NC stream of BS $i$. The signal-space of the $l$ th spatial NC stream of BS $i$ is $\mathbf{H}_{j, i} \mathbf{p}_{i, l}$ or $\mathbf{H}_{j, i} \mathbf{p}_{\tau}, \tau=I(i, l)$. According to the scaled signal-space alignment condition, there are some other spatial streams falls inside the same bin whose signal-spaces are identical to $\mathbf{H}_{j, i} \mathbf{p}_{\tau}$, i.e.,

$$
\begin{aligned}
& \widetilde{g}_{j, l^{\prime}}\left[\tau^{\prime}\right] \mathbf{f}_{j, l^{\prime}} \mathbf{H}_{j, I^{-1}(\tau)} \mathbf{p}_{\tau}=\widetilde{g}_{j, l^{\prime}}[\tau] \mathbf{f}_{j, l^{\prime}} \mathbf{H}_{j, I^{-1}\left(\tau^{\prime}\right)} \mathbf{p}_{\tau^{\prime}} \\
& \forall \tau^{\prime} \neq \tau, \quad\left(\tau, \tau^{\prime}\right) \in \mathcal{T}\left(\widetilde{\mathbf{g}}_{j, l^{\prime}}\right)
\end{aligned}
$$

after the receiver shaping. This signal is free of interference from other bins. Denote by

$\mathbf{q}_{j, l^{\prime}}=\widetilde{g}_{j, l^{\prime}}\left[\tau^{\prime}\right] \mathbf{f}_{j, l^{\prime}} \mathbf{H}_{j, I^{-1}(\tau)} \mathbf{p}_{\tau}=\widetilde{g}_{j, l^{\prime}}[\tau] \mathbf{f}_{j, l^{\prime}} \mathbf{H}_{j, I^{-1}\left(\tau^{\prime}\right)} \mathbf{p}_{\tau^{\prime}}$

Based on $\mathbf{q}_{j, l^{\prime}}$, User $j$ attempts to compute the following linear combination of the $\mathrm{NC}$ message sequences

$$
\mathbf{v}_{j, l^{\prime}}=\mathbf{g}_{j, l^{\prime}} \otimes \mathbf{U}
$$

by calculating

$$
\mathbf{q}_{j, l^{\prime}} \bmod \Lambda_{S}
$$

and quantize it to the nearest point in the fine lattice $\Lambda_{\tau}$, where $\Lambda_{\tau}$ is the finest lattice among those w.r.t. the nonzero entries of $\widetilde{\mathbf{g}}_{j, l^{\prime}}$. Here, for simplicity of presentation, the dithering operation as well as the minimum mean square error scaling are not shown here, and the details can be found in [18]. It can be shown that there exists a nested lattice code of rate $\widetilde{R}_{i, l}^{(j)}$, where the coarse lattice is simultaneously Rogers-good and Poltyrev-good while the fine lattices are Poltyrev-good [18], whose error probability of computing $\mathbf{v}_{j, l^{\prime}}$ vanishes as $n \rightarrow \infty$ as long as [18], [15]

$$
\begin{aligned}
& \widetilde{R}_{i, l}^{(j)}<\log _{2}\left(\left|\mathbf{f}_{j, l^{\prime}} \mathbf{H}_{j, i} \mathbf{p}_{\tau}\right|^{2} \rho\right)^{+} \log _{2} q \\
& \widetilde{R}_{i, I^{-1}\left(\tau^{\prime}\right)}^{(j)}<\log _{2}\left(\left|\mathbf{f}_{j, l^{\prime}} \mathbf{H}_{j, I^{-1}\left(\tau^{\prime}\right)} \mathbf{p}_{\tau^{\prime}}\right|^{2} \rho\right)^{+} \log _{2} q \\
& \forall \tau^{\prime} \neq \tau, \tau^{\prime} \in \mathcal{T}\left(\widetilde{\mathbf{g}}_{j, l^{\prime}}\right) .
\end{aligned}
$$

Taking the minimum of the rates $R_{i, l}^{(1)}, \ldots, R_{i, l}^{\left(K^{\prime}\right)}$ over all BSs, all NC messages sequences that are related to the $l$ th spatial stream of user $i$ can be reliably computed if

$\widetilde{R}_{i, l}<\min _{j \in\left\{1, \cdots, K^{\prime}\right\}} \widetilde{R}_{i, l}^{(j)}=\min _{j \in\left\{1, \cdots, K^{\prime}\right\}} \log _{2}\left(\left|\mathbf{f}_{j, l^{\prime}} \mathbf{H}_{j i} \mathbf{p}_{i, l}\right|^{2} \rho\right)^{+} \log _{2} q$. 
2) Achievable Message Rate:

Proposition 2: The following $L^{\prime}$-tuple of message rates is achievable

$$
\begin{aligned}
& R_{j} \leq \sum_{l^{\prime}=1}^{L_{j}^{\prime}} \min _{i, l: g_{i, l}\left[J\left(j, l^{\prime}\right)\right] \neq 0} \widetilde{R}_{i, l}, \\
& \sum_{i=1}^{K} \sum_{l=1}^{L_{i}} \widetilde{R}_{i, l}<\bar{C}_{F H} .
\end{aligned}
$$

given that $\mathbf{p}_{i, l}, l=1, \cdots, L_{i}, i=1, \cdots, K$, satisfy the signal-space alignment condition (36).

Proof. Consider the $l^{\prime}$ th data stream of User $j$. It is clear that those $\mathrm{NC}$ message sequences indexed by $i, l$ with $g_{i}^{l}\left[J\left(j, l^{\prime}\right)\right]=0$ are irrelevant to that data stream. In other words, the NC messages containing that data stream have indices $i, l: g_{i}^{l}\left[J\left(j, l^{\prime}\right)\right] \neq 0$. In addition, the message rate cannot be greater than the rate of the $\mathrm{NC}$ message that contains it. This leads to

$$
R_{j, l^{\prime}} \leq \min _{i, l: g_{i, l}\left[I\left(j, l^{\prime}\right)\right] \neq 0} \widetilde{R}_{i, l}
$$

Consider all data streams of User $j$. The rate is given in (53).

Next, recall that the FH consumption is given by $\sum_{i=1}^{K} \sum_{l=1}^{L_{i}} \widetilde{R}_{i, l}$. Thus, the rate in (47) is achievable if the FH capacity meets (54).

\section{B. Existence of Matrix $\mathbf{G}$ and and Signal-space Alignment Precoding}

In this part, we relax the presumed condition on aligned signal-spaces by investigating the existence of the NC generator matrix $\mathbf{G}$ and the feasibility of signal-space alignment.

In the previous section, we have seen that the $\mathrm{NC}$ generator matrix $\mathbf{G}$ determines the binning operation or the pattern of the signal-space alignment precoding. To accomplish the binning operation, there are some structural properties imposed on the NC generator matrix. These structural properties are similar to those studied in [20] for the uplink DMIMO, but the the difference is that they are imposed on the inverse NC generator matrix $\widetilde{\mathbf{G}}$, rather than the NC generator matrix as in [20]. To make this paper self-contained, the structural properties are summarized as follows.

Property 1: The number of rows of $\widetilde{\mathbf{G}}_{j}$, i.e. $L_{j}^{\prime}, j \in$ $\left\{1, \cdots, K^{\prime}\right\}$, is no greater than $N^{\prime}$.

Explanation: The number of rows of $\widetilde{\mathbf{G}}_{j}$ is the number of bins to be created at User $j$. For each user, the total receive signal-space has a dimension of $N^{\prime}$. As such, no more than $N^{\prime}$ bins can be created. Thus, $L_{j}^{\prime} \leq N^{\prime}$.

Define the Hamming weight of a vector as its number of non-zero entries.

Property 2: a) For BS $j$ such that $L_{j}^{\prime}=N^{\prime}$, the Hamming weight of a column of $\widetilde{\mathbf{G}}_{j}, j \in\left\{1, \cdots, K^{\prime}\right\}$, is 1 . (That is, each column of $\widetilde{\mathbf{G}}_{j}$ contains one non-zero entry.) Also, the sum of the Hamming weights of the rows of $\mathbf{G}_{j}$ is equal to $L$. b) For BS $j$ s.t. $L_{j}^{\prime}<N^{\prime}$, the Hamming weight of a column of $\widetilde{\mathbf{G}}_{j}, j \in\left\{1, \cdots, K^{\prime}\right\}$, is 1 or 0 . The sum of the Hamming weights of the rows of $\widetilde{\mathbf{G}}_{j}, j \in\left\{1, \cdots, K^{\prime}\right\}$, is no greater than $L$.

Explanation: a) This follows from that at each user, a spatial NC stream cannot be in two different bins, i.e., $\mathcal{B}_{j, l^{\prime}} \cap \mathcal{B}_{j, \bar{l}^{\prime}}=\emptyset$ for $\bar{l}^{\prime} \neq l^{\prime}$. Thus, If $L_{j}^{\prime}=N^{\prime}$, each column of $\widetilde{\mathbf{G}}_{j}$ contains one non-zero entry. In addition, the sum of the Hamming weights of the rows of $\widetilde{\mathbf{G}}_{j}$ is equal to $L$, as all $L$ spatial NC streams appears in the bins for signal-space alignment.

Property 3: The rank of $\widetilde{\mathbf{G}}$ is $L$ in $\mathrm{GF}(q)$.

We next present a proposition on the existence of $\widetilde{\mathbf{G}}$ satisfying all the above properties.

Lemma 1: There exists a NC generator matrix $\widetilde{\mathbf{G}}$ satisfying Properties 1-3 as long as the entries of $\widetilde{\mathbf{G}}$ belongs to $\{0,1, \ldots, p-1\}, p \geq 3$, for any $L \leq K^{\prime} N^{\prime}$. (See proof in [20], Appendix.)

Remark 2: Lemma 1 suggests that we can construct the matrix $\widetilde{\mathbf{G}}$ with entries only in $\{0,1,2\}$.

We next investigate the existence of the precoding matrice that satisfy the signal-space alignment condition w.r.t. $\widetilde{\mathbf{G}}$. Let $\mathbf{W}$ be a matrix composed in a block-wise fashion as follows. The first $N^{\prime}$-rows are given by

$$
\left[\mathbf{0}, \ldots, g_{1}^{1}\left(\tau^{\prime}\right) \mathbf{H}_{1, I^{-1}(\tau)}, \mathbf{0}, \ldots, g_{1}^{1}(\tau) \mathbf{H}_{1, I^{-1}\left(\tau^{\prime}\right)}, \mathbf{0}, \ldots, \mathbf{0}\right] .
$$

Likewise, consider other events of $\left(\tau, \tau^{\prime}\right) \in \mathcal{T}\left(\mathbf{g}_{1,1}\right)$, the following several $N^{\prime}$-rows of $\mathbf{W}$ can be obtained. Repeating the above process for all $j=1, \cdots, K^{\prime}, l^{\prime}=1, \cdots, L^{\prime}$, the matrix $\mathbf{W}$ is obtained. With $\mathbf{W}$, the criterion of the scaled signal-space alignment becomes

$$
\mathbf{W} \boldsymbol{\eta}=\mathbf{0}
$$

where

$$
\boldsymbol{\eta}=\left[\mathbf{p}_{1,1}^{T}, \cdots, \mathbf{p}_{1, L_{1}}^{T}, \mathbf{p}_{2,1}^{T}, \cdots, \mathbf{p}_{K, L_{K}}^{T}\right]^{T} .
$$

Then, the signal-space alignment is feasible if $\mathbf{W}$ is not overdetermined, i.e., the rank of $\mathbf{W}$ is smaller than the dimension of $\boldsymbol{\eta}$.

The feasibility of signal-space alignment is presented in the following proposition.

Proposition 3: For a given NC generator matrix $\widetilde{\mathbf{G}}$ satisfying Property 1-3, the corresponding signals-space alignment criterion can be satisfied if $L$ satisfies

$$
N>\frac{L-N^{\prime}}{L} \overline{K^{\prime}} N^{\prime}, \overline{K^{\prime}}=\left\lceil\frac{L}{N^{\prime}}\right\rceil .
$$

Proof. First consider that $\overline{K^{\prime}}=K^{\prime}$. It can be shown that the dimension of $\widetilde{\mathbf{H}}$ is $\left(L-N^{\prime}\right) N^{\prime} K^{\prime}$ by $L N$. To ensure that $\widetilde{\mathbf{H}}$ matrix is strictly not over-determined, the following must be satisfied

$$
L N>\left(L-N^{\prime}\right) K^{\prime} N^{\prime}
$$

which yields Eq. (59). Then, there exists at least one solution which satisfies the signal-space alignment criterion in (36). Consider next $\overline{K^{\prime}}=\left\lceil\frac{L}{N^{\prime}}\right\rceil<K^{\prime}$. In such cases, $\overline{K^{\prime}}$ active BSs 
are sufficient for generating the $L \mathrm{NC}$ message sequences in the proposed scheme. The condition to ensure that $\widetilde{\mathbf{H}}$ matrix is not over-determined for a $\overline{K^{\prime}}$-BS system is simply (60).

\section{Achievable Rate of the Proposed Scheme}

Combining Lemma 1 and Propositions 2 and 3, we have the following result.

Theorem 1: The following $L^{\prime}$-tuple of message rates is achievable

$$
\begin{aligned}
& R_{j} \leq \sum_{l^{\prime}=1}^{L_{j}^{\prime}} \min _{i, l: g_{i, l}\left[J\left(j, l^{\prime}\right)\right] \neq 0} \widetilde{R}_{i, l}, \\
& \widetilde{R}_{i, l}<\min _{j \in\left\{1, \cdots, K^{\prime}\right\}} \log _{2}\left(\left|\mathbf{f}_{j, l^{\prime}} \mathbf{H}_{j, i} \mathbf{p}_{i, l}\right|^{2} \rho\right)^{+} \log _{2} q, \\
& l \in\left\{1, \cdots, L_{i}\right\}, i \in\{1, \cdots, K\}, \\
& \sum_{i=1}^{K} \sum_{l=1}^{L_{i}} \widetilde{R}_{i, l}<\bar{C}_{F H} .
\end{aligned}
$$

with $\mathbf{p}_{i, l}$ satisfying the condition in (36), if the total number of spatial NC streams $L$ satisfies

$$
L<\frac{\left(N^{\prime}\right)^{2} \bar{K}^{\prime}}{\bar{K}^{\prime} N^{\prime}-N}, \bar{K}^{\prime}=\left\lceil\frac{L}{N^{\prime}}\right\rceil .
$$

\section{Discussions}

1) Equal Gain Signal-space Alignment: Previously, we have shown that a matrix $\widetilde{\mathbf{G}}$ satisfying Properties 1-3 exists for any $L \leq K^{\prime} N^{\prime}$. It is interesting to note that a matrix $\widetilde{\mathbf{G}}$ with entries in $\{0,1,2\}$ is sufficient for the existence. Using such a matrix, from (36), the ratio between the norms of two aligned spatial NC streams are either 1 or 2 . We next consider the equal gain signal-space alignment (37) where the entries of $\widetilde{\mathbf{G}}$ can only be in $\{0,1\}$.

Lemma 2: There exists an $L \times L$ matrix $\widetilde{\mathbf{G}}$ with binary entries in $\{0,1\}$ satisfying Properties $1-3$ if and only if at most one value among $L_{1}^{\prime} \cdots L_{K^{\prime}}^{\prime}$ is equal to $N^{\prime}$. This is equivalently to say that

$$
L \leq\left(K^{\prime}-1\right)\left(N^{\prime}-1\right)+N^{\prime}=K^{\prime}\left(N^{\prime}-1\right)+1 .
$$

Proof. Without loss of generality, assume that $L_{1}^{\prime} \geq L_{2}^{\prime} \cdots \geq$ $L_{K^{\prime}}^{\prime}$. Let $\mathbf{G}$ be an $L \times L \mathrm{NC}$ generator matrix over $\mathrm{GF}(2)$ satisfying Properties 1-3. Consider the case $N^{\prime}=L_{1}^{\prime}=L_{2}^{\prime}$. As a consequence of Property 2, the sum of the first $2 N^{\prime}$ row vectors in $\mathbf{G}$ will yield a zero vector over $\mathrm{GF}(2)$, which implies $\operatorname{rank}(\mathbf{G})<L$, a contradiction to Property 3. Thus, $N^{\prime}>L_{2}^{\prime}$ and necessity part is proved. For the sufficiency part, define the matrix $\mathbf{G}$ in the same way as the construction in the second paragraph of Proposition 1. Following exactly the same argument therein, the considered $\mathbf{G}$ over $\mathrm{GF}(2)$ is a desired generator matrix either for the case $N^{\prime}=L_{1}^{\prime}>L_{2}^{\prime} \geq$ $\cdots \geq L_{K^{\prime}}$ or $N^{\prime}>L_{1}^{\prime} \geq L_{2}^{\prime} \geq \cdots \geq L_{K^{\prime}}$.

Combining Lemma 2 and Proposition 3, we have the following corollary.
Corollary 1: There exists a matrix $\widetilde{\mathbf{G}}$ with entries of $\{0,1\}$ such that the signal-space alignment can be met if

$$
L \leq \min \left(K^{\prime}\left(N^{\prime}-1\right)+1, \frac{\left(N^{\prime}\right)^{2} \bar{K}^{\prime}}{\bar{K}^{\prime} N^{\prime}-N}\right)
$$

where $\bar{K}^{\prime}=\left\lceil\frac{L}{N^{\prime}}\right\rceil$.

For most configurations of interests, (66) is dominated by the second term. These suggests that equal gain signal-space alignment is sufficient for most configurations in practice.

2) Relation to Interference Alignment: Our proposed scheme boils down to the IA strategy if we choose the inverse NC generator matrix $\widetilde{\mathbf{G}}$ to be some concatenated version of a number of identity matrices. Therefore, the acheivable rate and DoF of our proposed scheme are no lower than that of the IA scheme in the DMIMO setting. It can be expected that by choosing a better $\widetilde{\mathbf{G}}$, improved performance over that with IA can be achieved. This claim holds even when symbolextension over time-varying channels is applicable.

3) Where Does the Gain Come From?: It is interesting to note that while PNC is essentially performing "structured binning" over a $n$-dimensional superimposed constellation [15], the proposed signal-space alignment performs binning over the spatial streams in $N^{\prime}$ dimension receive signal spaces according to a carefully designed $\widetilde{\mathbf{G}}$ matrix. These two components are coupled together, combining which yields an overall gain in achievable rate or DoF. The strategy using IA ignores the potential coding gain that can be achieved by PNC in the DMIMO system, thus is expected to be outperformed by our proposed scheme.

\section{Downlink DMimo with Two And Three BAse STATIONS}

This section studies the D-MIMO systems with $K=2$ and $3 \mathrm{BSs}$, where we focus on characterizing the DoF. We restrict our discussions to the symmetric configuration with $K=K^{\prime}$.

\section{A. Downlink D-MIMO with $K=2 \mathrm{BSS}$}

We first present a result on the DoF of the downlink DMIMO scheme with $K=2$.

Theorem 2: Let the $\mathrm{FH}$ capacity be given by $C_{F H}=$ $L \log \rho+\epsilon$, where $\epsilon=o(1)$. For $L \leq \min \left(2 N, 2 N^{\prime}\right)-1$, the total DoF of the downlink D-MIMO with $K=2 \mathrm{BSs}$ is given by

$$
d=L
$$

Proof. We first consider the achievability. Using Proposition 3, it can be shown that (59) is met for any $L \leq$ $\min \left(2 N, 2 N^{\prime}\right)-1$. Thus, the signal-space alignment criterion in (36) is guaranteed. In addition, from Lemma 1, a NC generator matrix G satisfying Properties 1-3 exists for $q \geq 2$. Thus, the rate in Proposition 2 is achievable. The corresponding sum-rate is given by

$$
\sum_{i=1}^{K} \sum_{l=1}^{L_{i}} \min _{j \in\left\{1, \cdots, K^{\prime}\right\}} \log _{2}\left(\left|\mathbf{f}_{j, l^{\prime}} \mathbf{H}_{j, i} \mathbf{p}_{i, l}\right|^{2} \rho\right)^{+} \log _{2} q .
$$


Note that $\mathbf{f}_{j, l^{\prime}} \mathbf{H}_{j, i}$ has non-vanishing values in probability. As $\rho \rightarrow \infty$, the DoF is $\sum_{i=1}^{K} L_{i}=L$.

The converse follows directly from that max-flow min-cut theorem for a general network. Due to the rate-constrained $\mathrm{FH}$, the maximum information rate is no more than $L \log \rho+$ $o(1)$.

Note that as $N \rightarrow \infty$, as long as $C_{F H}$ scales like $\min \left(2 N, 2 N^{\prime}\right)$, the downlink D-MIMO with two BSs asymptotically achieves a DoF of $\min \left(2 N, 2 N^{\prime}\right)$. This is equivalent to the DoF upper bound given by a MIMO broadcast channel with $2 N$ co-located transmit antennas.

An example of the proposed distributed MIMO broadcasting scheme with $N=3$ is shown below, where a DoF of $L=2 N-1$ is achieved. Consider that User 1 and 2 have $L_{1}^{\prime}=3$ and $L_{2}^{\prime}=2$ data streams, respectively. The entries of the data streams are drawn i.i.d. from $\operatorname{GF}(q)$. Consider that the $\mathrm{NC}$ generator matrix being

$$
\mathbf{G}=\left[\begin{array}{ccccc}
1 & 0 & 1 & q-1 & 0 \\
1 & 1 & 1 & q-1 & q-1 \\
0 & 0 & 1 & 0 & 0 \\
0 & 0 & q-1 & 1 & 0 \\
q-1 & 0 & q-1 & 1 & 1
\end{array}\right]
$$

which is used for pre network coding over the 5 data streams. The resultant $\mathrm{NC}$ messages streams $\mathbf{U}$ are forwarded to the two BSs via the FH links. If $C_{F H}=5 \log \rho+\epsilon$, these $\mathrm{NC}$ messages can be reliably delivered to the BSs, for a sufficiently large $\epsilon=o(1)$.

The matrix $\mathbf{G}$ has a full rank 5 in $\mathrm{GF}(q)$. The corresponding inverse NC generator matrix is

$$
\widetilde{\mathbf{G}}=\left[\begin{array}{c}
\widetilde{\mathbf{G}}_{1} \\
\widetilde{\mathbf{G}}_{2}
\end{array}\right]=\mathbf{G}^{-1}=\left[\begin{array}{ccccc}
1 & 0 & 0 & 1 & 0 \\
0 & 1 & 0 & 0 & 1 \\
0 & 0 & 1 & 0 & 0 \\
0 & 0 & 1 & 1 & 0 \\
1 & 0 & 0 & 0 & 1
\end{array}\right]
$$

We next perform signal-space alignment based on the pattern given by $\widetilde{\mathbf{G}}$. Form Eq. (56), the corresponding $\mathbf{W}$ matrix for the constraints for the signal-space alignment is given by

$$
\mathbf{W}=\left[\begin{array}{ccccc}
\mathbf{H}_{1,1} & \mathbf{0} & \mathbf{0} & \mathbf{H}_{1,2} & \mathbf{0} \\
0 & \mathbf{H}_{1,1} & \mathbf{0} & \mathbf{0} & \mathbf{H}_{1,1} \\
\mathbf{0} & \mathbf{0} & \mathbf{H}_{2,1} & \mathbf{H}_{2,2} & \mathbf{0} \\
\mathbf{H}_{2,1} & \mathbf{0} & \mathbf{0} & \mathbf{0} & \mathbf{H}_{2,2}
\end{array}\right]
$$

The conditions for the signal alignment w.r.t. the given $\widetilde{\mathbf{G}}$ matrix is

$$
\mathbf{W}\left[\mathbf{p}_{1,1}^{T}, \mathbf{p}_{1,2}^{T}, \mathbf{p}_{1,3}^{T}, \mathbf{p}_{2,1}^{T}, \mathbf{p}_{2,2}^{T}\right]^{T}=\mathbf{0} .
$$

Since the condition on Proposition 3 is met for this case, a solution on the precoding matrices does exist which ensures the signal-space alignment with the pattern given by $\widetilde{\mathbf{G}}$.

The two BS simultaneously broadcast their spatial NC streams. With the precoding matrices obtained from (71), at User 1, we obtain

$$
\mathbf{H}_{1,1} \mathbf{p}_{1,1}=-\mathbf{H}_{1,2} \mathbf{p}_{2,1} \text { and } \mathbf{H}_{1,1} \mathbf{p}_{1,2}=-\mathbf{H}_{1,2} \mathbf{p}_{2,2} \text {. }
$$

At User 1, the signal-space of the first spatial stream of BS 1 and that of the first spatial stream of BS 2 are aligned.
Meanwhile, the second stream of BS 1 and the second stream of BS 2 are aligned. The signal-space of the third spatial stream of BS 1 is $\mathbf{H}_{1,1} \mathbf{p}_{1,3}$.

Next, using the receiver shaping (38), the received signal $\mathbf{Y}_{1}$ is projected to the subspace that is perpendicular to the subspace $\operatorname{span}\left\{\mathbf{H}_{1,1} \mathbf{p}_{1,2}, \mathbf{H}_{1,1} \mathbf{p}_{1,3}\right\}$. The resultant signal is free of interference from other signals streams in $\operatorname{span}\left\{\mathbf{H}_{1,1} \mathbf{p}_{1,2}, \mathbf{H}_{1,1} \mathbf{p}_{1,3}\right\}$. Based on that, post PNC is used to compute the first linear combination of the $\mathrm{NC}$ message sequences

$$
\mathbf{v}_{1,1}=\widetilde{\mathbf{g}}_{1,1} \otimes \mathbf{U}=\mathbf{u}_{1,1} \oplus \mathbf{u}_{2,1} .
$$

Using the $\mathbf{G}$ matrix in (68), it can be easily verify that

$$
\begin{aligned}
\mathbf{v}_{1,1} & =\mathbf{u}_{1,1} \oplus \mathbf{u}_{2,1} \\
& =\mathbf{b}_{1,1} \oplus \mathbf{b}_{1,3} \oplus(q-1) \mathbf{b}_{2,1} \oplus(q-1) \mathbf{b}_{1,3} \oplus \mathbf{b}_{2,1} \\
& =\mathbf{b}_{1,1}
\end{aligned}
$$

which is the desired (first) data stream of User 1.

Similarly, the received signal $\mathbf{Y}_{1}$ is projected to the subspace that is perpendicular to the subspace $\operatorname{span}\left\{\mathbf{H}_{1,1} \mathbf{p}_{1,1}, \mathbf{H}_{1,1} \mathbf{p}_{1,3}\right\}$, and the desired (second) data stream of User 1

$$
\mathbf{v}_{1,2}=\widetilde{\mathbf{g}}_{1,2} \otimes \mathbf{U}=\mathbf{u}_{1,2} \oplus \mathbf{u}_{2,2}=\mathbf{b}_{1,2}
$$

is computed using post PNC. The received signal $\mathbf{Y}_{1}$ is projected to the subspace that is perpendicular to the subspace $\operatorname{span}\left\{\mathbf{H}_{1,1} \mathbf{p}_{1,1}, \mathbf{H}_{1,1} \mathbf{p}_{1,2}\right\}$, and the message

$$
\mathbf{v}_{1,3}=\widetilde{\mathbf{g}}_{1,3} \otimes \mathbf{U}=\mathbf{b}_{1,3}
$$

is decoded. Likewise, at BS 2, the linear combinations of the $\mathrm{NC}$ message sequences

$$
\mathbf{v}_{2,1}=\widetilde{\mathbf{g}}_{2,1} \otimes \mathbf{U}=\mathbf{b}_{2,1} \text { and } \mathbf{v}_{2,2}=\widetilde{\mathbf{g}}_{2,2} \otimes \mathbf{U}=\mathbf{b}_{2,2}
$$

are computed which are equal to the desired data streams. The sum DoF is 5 .

Let us consider two benchmark schemes for comparison purpose. The first one is the orthogonalized transmission, where the two BSs take turn to transmit. The second one is the a scheme which directly applies the strategy for a interference channel, where User 1 only decodes the BS 1's data streams while User 2 only decodes the BS 2's data streams. For the D-MIMO setting with $K=K^{\prime}=2$, the DoFs of both benchmarks are equal to $N$ if the FH capacity scales like $C_{F H} \propto N \log \rho$. Yet, as $C_{F H}$ scales at a higher speed, i.e., $C_{F H} \propto L \log \rho, L>N$, none of them can yield increased DoF. In contrast, our proposed yields a DoF of $L$ for $N<L \leq 2 N-1$. Therefore, our proposed achieves up to $\frac{2 N-1}{N}$ times of DoF of the benchmark schemes.

Fig. 3 shows the averaged achievable sum-rates of the proposed scheme for a D-MIMO system of $K=K^{\prime}=2$, $N=M=3$. Rayleigh fading are considered in the simulations, and the results are obtained by averaging a large number of fading realizations. Here, the FH capacity is given by ${ }^{5} C_{F H}=L \log \rho+\epsilon, L=2,3,4,5$. The achievable rate of

\footnotetext{
${ }^{5}$ A sufficiently large $\epsilon=o(1)$ is considered.
} 


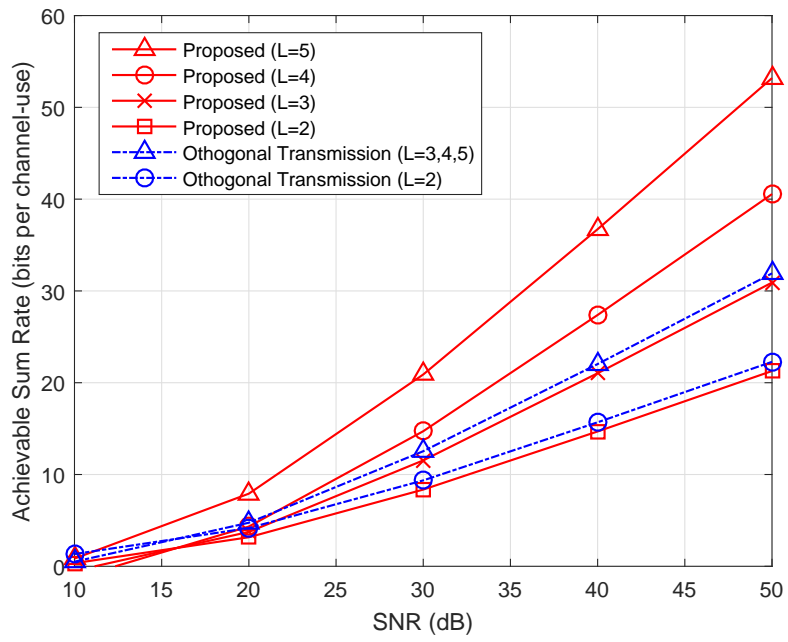

Fig. 3. Achievable sum-rate of the proposed distributed MIMO broadcasting scheme with $K=K^{\prime}=2, N=N^{\prime}=3$.

the benchmark scheme using orthogonal transmission (with zero-forcing precoding) is also plotted. It is demonstrated that the proposed scheme can achieve a much greater sumrate over the benchmark scheme (even with infinite symbolextension). The performance gain is primarily due to the overwhelmingly higher DoF.

Remark 3: In the case studied above, equal gain signal space alignment, where the entries of $\widetilde{\mathbf{G}}$ belong to $\{0,1\}$, is sufficient to guarantee the DoF result in Theorem 2. Such an equal gain signal space alignment scheme is easier to implement in practice than the non-equal gain signal-space alignment scheme, as the precise scaling is difficult to be guaranteed.

Remark 4: In this paper, no efforts are made on optimizing the directions of the bins and the power profile. A joint optimization of the NC generator matrix and precoding matrices $(\widetilde{\mathbf{G}}, \mathbf{P})$ can yield further power gain, but is unlikely to yield further DoF gain. Nevertheless, the joint optimization of $(\widetilde{\mathbf{G}}, \mathbf{P})$ could be an interesting future work.

We would like to note that the proposed scheme is targeting at the high SNR regime where achieving a high DoF plays the key role. It is demonstrated that our proposed scheme significantly outperforms the benchmark interference alignment scheme and orthogonal transmission scheme at the high SNR regime and some practical SNR values.

We next compare the numerical results of our proposed scheme and the compress-and-forward based scheme in [32]. In simulating the performance of [32], we consider linear precoding at the $\mathrm{CU}$ using regularized channel inversion (or MMSE) algorithm and joint compression at the distributed BSs. We consider that there are two BSs and two user nodes where each BS and user node are equipped with three antennas, i.e., $K=K^{\prime}=2, N=N^{\prime}=3$. We consider fixed SNR values of 20 and $30 \mathrm{dBs}$ and plot their performance, respectively. The figures are shown in Fig. 4 and Fig. 5. For $\mathrm{SNR}=20 \mathrm{~dB}$, it is observed that our proposed scheme con-

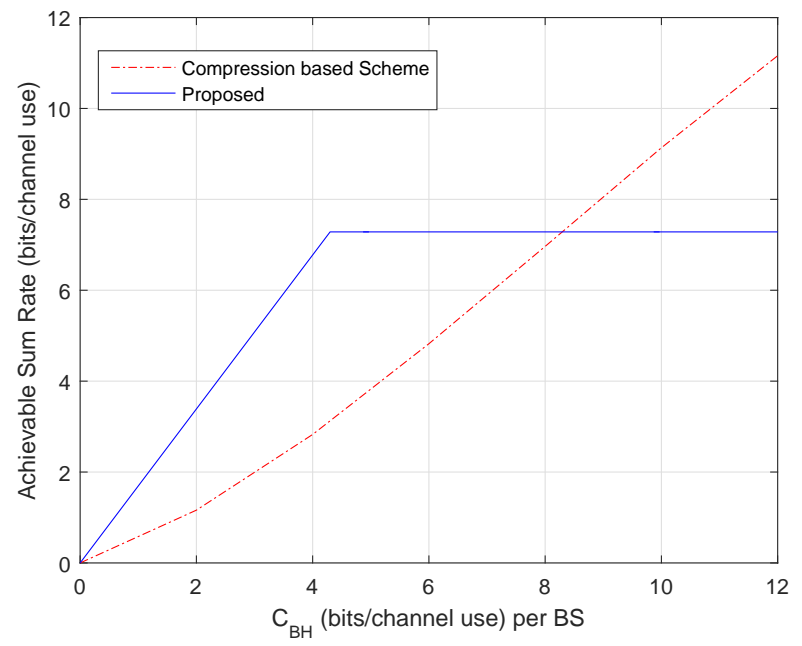

Fig. 4. Comparison of achievable sum rates of the proposed scheme and [32], where $K=K^{\prime}=2, N=N^{\prime}=3$, and $\mathrm{SNR}=20 \mathrm{~dB}$

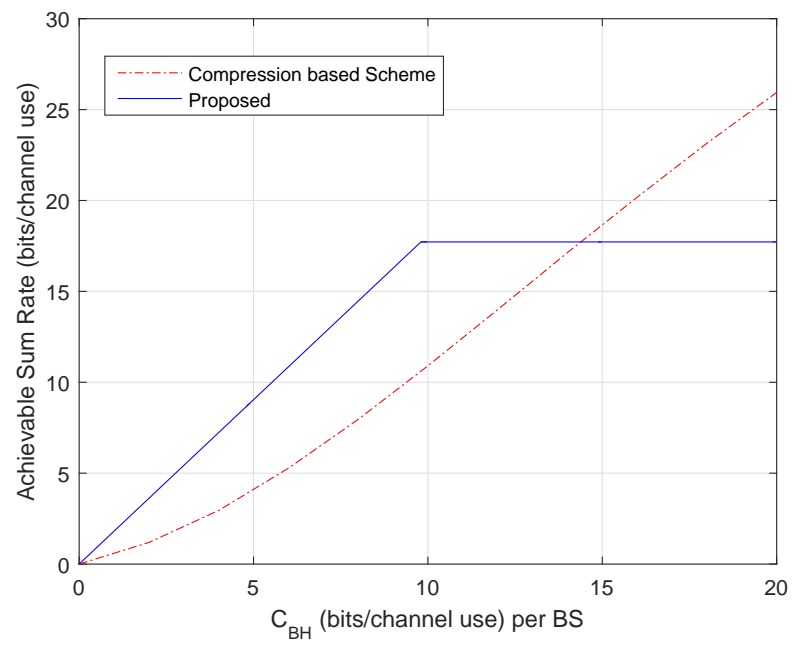

Fig. 5. Comparison of achievable sum rates of the proposed scheme and [32], where $K=K^{\prime}=2, N=N^{\prime}=3$, and $\mathrm{SNR}=30 \mathrm{~dB}$

siderable outperforms the compression based scheme in [32] for the low-to-moderate FH capacity range. For the ample FH regime, e.g., where the sum fronthaul rate consumption is more than twice of the sum rate, the proposed scheme has lower sum rate than the compression based scheme in [32]. This result for the multi-antenna configuration agrees with the single-antenna case shown in [32] where reverse CF outperforms the compression based scheme for the low-tomoderate $\mathrm{FH}$ range. For $\mathrm{SNR}=30 \mathrm{~dB}$, similar behaviors are observed.

\section{B. An Example for Downlink D-MIMO with $K=3 \mathrm{BSs}$}

In this part, we consider a downlink D-MIMO with $K=$ $K^{\prime}=3$. Here we study a case where a DoF of $L=7$ for $N^{\prime}=3$ is achieved using our proposed scheme. Consider that there are 3 data streams for User 1, and 2 data streams 
for each of users 2 and 3. The inverse NC generator matrix is constructed as follows. Consider equal gain signal space alignment that the entries of $\widetilde{\mathbf{G}}$ belong to $\{0,1\}$. First consider a matrix consists of various 3 by 3 permutation matrices $\Pi_{3}$ and 2 by 2 permutation matrices $\Pi_{2}$, with proper zeropadding. An example is given below:

$$
\widetilde{\mathbf{G}}^{\prime}=\left[\begin{array}{lllllll}
1 & 0 & 0 & 1 & 0 & 1 & 0 \\
0 & 1 & 0 & 0 & 1 & 0 & 0 \\
0 & 0 & 1 & 0 & 0 & 0 & 1 \\
0 & 1 & 0 & 0 & 1 & 0 & 1 \\
1 & 0 & 0 & 1 & 0 & 0 & 0 \\
0 & 0 & 1 & 0 & 0 & 1 & 0 \\
0 & 0 & 1 & 1 & 0 & 0 & 1 \\
1 & 0 & 0 & 0 & 1 & 0 & 0 \\
0 & 1 & 0 & 0 & 0 & 1 & 0
\end{array}\right]
$$

which has full rank 7 in $\operatorname{GF}(q)$ for any $q \gtrsim 2$. In particular, only 7 rows are needed in constructing $\widetilde{\mathbf{G}}$. We choose by expurgating the sixth and seventh row of $\widetilde{\mathbf{G}}^{\prime}$, obtaining

$$
\widetilde{\mathbf{G}}=\left[\begin{array}{lllllll}
1 & 0 & 0 & 1 & 0 & 1 & 0 \\
0 & 1 & 0 & 0 & 1 & 0 & 0 \\
0 & 0 & 1 & 0 & 0 & 0 & 1 \\
0 & 1 & 0 & 0 & 1 & 0 & 1 \\
1 & 0 & 0 & 1 & 0 & 0 & 0 \\
1 & 0 & 0 & 0 & 1 & 0 & 0 \\
0 & 1 & 0 & 0 & 0 & 1 & 0
\end{array}\right]
$$

which has a full rank of 7 . The NC generator matrix is

$\mathbf{G}=\widetilde{\mathbf{G}}^{-1}=\left[\begin{array}{ccccccc}q-1 & q-1 & 0 & 0 & 1 & 1 & 1 \\ q-1 & 0 & 0 & 0 & 1 & 0 & 1 \\ 0 & 1 & 1 & q-1 & 0 & 0 & 0 \\ 1 & 1 & 0 & 0 & 0 & q-1 & q-1 \\ 1 & 1 & 0 & 0 & q-1 & 0 & q-1 \\ 1 & 0 & 0 & 0 & q-1 & 0 & 0 \\ 0 & q-1 & 0 & 1 & 0 & 0 & 0\end{array}\right.$

The corresponding $\mathbf{W}$ matrix for the signal-space alignment constraints can be obtained. A solution for the signal-space alignment exists if the condition in Proposition 3 is met, which is $7 N>12 N^{\prime}$. That is, more BS antenna is required to realize the DoF of 7 in this example.

Let us first discuss two benchmark schemes for $N^{\prime}=3$. For the orthogonal transmission, the DoF is just 3. For the interference channel strategy, it is well-known that interference alignment (IA) can yield a greater DoF. Using symbol extension for a time-varying channel, a DoF of 4.5 can be achieved using IA. However, if the FH scales like $C_{F H} \propto L \log \rho, L>4.5$, the DoF using the IA strategy is still 4.5. Thus, our proposed has greater DoF than IA and orthogonal transmission. Fig. 6 shows the averaged achievable sum-rates of the proposed scheme for a downlink D-MIMO of $K=K^{\prime}=3, N=6, N^{\prime}=3$. Here, the FH capacity is given by $C_{F H}=L \log \rho+\epsilon, L=2, \ldots, 7$. The rate of the benchmark scheme using orthogonal transmission and IA in [28] are also plotted for comparison. It is demonstrated that the proposed scheme can achieve a considerably greater sum-rate over the two benchmark schemes, due to the overwhelmingly higher DoF. At a SNR of $50 \mathrm{~dB}$, the proposed scheme has a throughput gain of about $70 \%$ over the benchmark scheme.

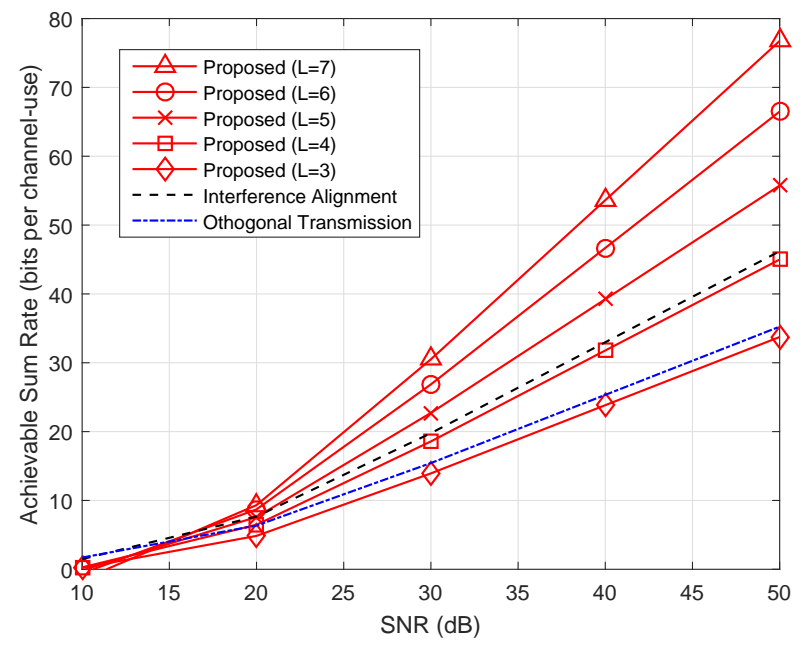

Fig. 6. Achievable sum-rate of the proposed distributed MIMO broadcasting scheme with $K=K^{\prime}=3, N=6, N^{\prime}=3$.

\section{CONCLUSIONS AND FUTURE WORKS}

A new network coding based downlink distributed MIMO broadcasting scheme, using reverse compute-and-forward and signal space alignment precoding, is proposed. A NC generator matrix is first designed and employed for pre network coding of the users' messages. The network coded messages are forwarded from the central unit to the distributed BSs. At each user node, post physical-layer network coding is employed which reverses (or equalizes) the pre network coding and recovers the desired messages. Our proposed signal-space alignment method provides a bridging between these two network coding operations. An achievable information rate of the proposed scheme is derived based on the existence of NC generator matrix, signal-space alignment precoding matrices, and "good" nested lattice codes. It is demonstrated that our proposed scheme has a greater DoF over the interference alignment strategy. Our proposed scheme also outperforms the compress-and-forward scheme that utilizes joint compression of the precoded signals to meet the fronthaul rate constraint, at the low-to-medium front-haul capacity regime.

This paper paves avenue for several interesting future works. It remains open whether symbol-extension can further improve the DoF of the proposed scheme, for time-varying channels. Although our proposed scheme brings about DoF gain, the supreme of DoF of the D-MIMO for a given front-haul capacity remains unsolved. In addition, it remains unknown whether the different choice of NC generator matrix $\mathbf{G}$ will have a great impact on the performance. Thus, how to jointly optimize the $\mathrm{NC}$ generator matrix $\mathbf{G}$ and the precoding matrices remains open. Finally, the impact of the physical-layer network coding based approach for the Fog radio network with local caching is an interesting question. It is believed that these aspects deserve further research efforts. 


\section{REFERENCES}

[1] C. Bernardos, A. D. Domenico, J. Ortin, P. Rost, and D. Wübben, "Challenges of designing jointly the backhaul and radio access network in a cloud-based mobile network," in Future Network \& Mobile Summit 2013, Lisbon, Portugal,, Jul 2013.

[2] M. HadZialic, B. Dosenovic, M. Dzaferagic, and J. Musovic, "CloudRAN: Innovative radio access network architecture," ELMAR, 2013 55th International Symposium, pp. 115-120, 2013.

[3] C. Mobile, "C-RAN: the road towards green RAN," White Paper, ver 2.5, China Mobile Research Institute, Oct. 2011.

[4] Y. Zhou and W. Yu, "Optimized backhaul compression for uplink cloud radio access network," IEEE Jour. Selected Area in Comm., vol. 32, no. 6, pp. 1295-1307, June 2014.

[5] D. Lee, H. Seo, B. Clerckx, E. Hardouin, D. Mazzarese, S. Nagata, and K. Sayana, "Coordinated multipoint transmission and reception in lte-advanced: deployment scenarios and operational challenges," IEEE Communications Magazine, vol. 50, no. 2, pp. 148-155, 2012.

[6] M. G. S. Chia and P. Brick, "The next challenge for cellular networks: backhaul," IEEE Microw. Mag., vol. 10, no. 5, pp. 54-66, Aug. 2009.

[7] S. Z. O. Tipmongkolsilp and A. Jukan, "The evolution of cellular backhaul technologies: current issues and future trends," IEEE Commun. Surveys Tuts., vol. 13, no. 1, pp. 97-113, 2011.

[8] Q. Zhang, C. Yang, and A. Molisch, "Downlink base station cooperative transmission under limited-capacity backhaul," IEEE Trans. Wireless Comm., vol. 12, no. 8, pp. 3746-3759, 2013.

[9] M. K. Karakayali, G. J. Foschini, and R. A. Valenzuela, "Network coordination for spectrally efficient communications in cellular systems," IEEE Wireless Commun. Mag., vol. 13, no. 4, pp. 56-61, Aug. 2006.

[10] D. Gesbert, S. Hanly, H. Huang, S. Shamai, O. Simeone, and W. Yu, "Multi-cell MIMO cooperative networks: a new look at interference," IEEE Jour. Sel. Area. Comm., vol. 28, no. 9, pp. 1380-1408, Dec. 2010.

[11] O. Simeone, "Distributed MIMO in multi-cell wireless systems via finite-capacity links," in Proc. ISCCSP, 2008.

[12] O. Simeone, O. Somekh, H. V. Poor, and S. Shamai, "Local base station cooperation via finite-capacity links for the uplink of linear cellular networks," IEEE Trans. Inf. Theory., vol. 55, no. 1, pp. 190-204, Jan. 2009.

[13] S.-Y. R. Li, Q. T. Sun, and Z. Shao, "Linear network coding: theory and algorithms," Proc. IEEE, vol. 99, no. 3, pp. 372-387, Mar. 2011

[14] T. Yang, Q. T. Sun, A. Zhang, and J.Yuan, "A linear network coding approach for uplink distributed MIMO systems : protocol and outage behavior," IEEE Journal on Selected Areas Comm., vol. 33, no. 2, pp. 250-263, Feb. 2015.

[15] B. Nazer and M. Gastpar, "Compute-and-forward: Harnessing interference through structured codes," IEEE Trans. Inf. Theory, vol. 57, no. 10, pp. 6463-6486, Oct. 2011.

[16] S. Zhang, S. Liew, and P. P. Lam, "Hot topic: physical-layer network coding," Proceedings of the 12th annual international conference on Mobile computing and networking, pp. 358-365, 2006.

[17] T. Yang and I. B. Collings, "On the optimal design and performance of linear physical-layer network coding for fading two-way relay channels," IEEE Trans. Wireless Comm., vol. 13, no. 2, pp. 956-967, Feb. 2014

[18] W. Nam, S. Chung, and Y. H. Lee, "Capacity of the gaussian twoway relay channel to within 1/2 bit," IEEE Trans. Inf. Theory, vol. 56, no. 11, pp. 5488-5494, Nov. 2010.

[19] J.-B. L. N. Lee and J. Chun, "Degrees of freedom of the MIMO Y channel: signal space alignment for network coding," IEEE Trans. Inf. Theory, vol. 56, no. 7, pp. 3332-3342, July. 2010.

[20] T. Yang, X. Yuan, and Q. Sun, "A signal-space aligned network coding approach to distributed MIMO," Submitted to IEEE Trans. Signal Proc., 2015.

[21] V. Ntranos, V. Cadambe, B. Nazer, and G. Caire, "Integer-forcing interference alignment," IEEE Int. Symp. Inf. Theory, pp. 574-578, 2013.

[22] R. Wang, X. Yuan, and M. Tao, "Degrees of freedom of MIMO multiway relay channel with clustered pairwise exchange," IEEE $J$. Sel. Areas Commun., vol. 33, no. 2, pp. 337-351, Feb. 2015.

[23] Z. Fang, X. Wang, and X. Yuan, "Beamforming design for multiuser two-way relaying: A unified approach via max-min SINR," IEEE Trans. Signal Processing, vol. 61, no. 23, pp. 5841-5852, Dec. 2013.

[24] R. Zakhour and S. Hanly, "Base station cooperation on the downlink: Large system analysis," IEEE Trans. Inf. Theory., vol. 58, no. 4, pp. 2079-2106, Apr. 2012.
[25] S.-N. Hong and G. Caire, "Compute-and-forward strategies for cooperative distributed antenna systems," IEEE Trans. Inf. Theory, vol. 59, no. 9 , pp. 5227-5243, Sep. 2013.

[26] P. Marsch, "Coordinated multi-point under a constrained backhaul and imperfect channel knowledge," VOGT, 2010.

[27] C. M. Yetis, T. Gou, S. A. Jafar, and A. H. Kayran, "On feasibility of interference alignment in MIMO interference networks," IEEE Trans. Signal Proc., vol. 58, no. 9, pp. 4771-4782, Sept. 2010.

[28] V. Cadambe and S. Jafar, "Interference alignment and the degrees of freedom of the $\mathrm{K}$ user interference channel," IEEE Trans. Inf. Theory, vol. 54, no. 8, pp. 3425-3441, Aug. 2008.

[29] L. Yang, T. Yang, J.Yuan, and P. An, "Achieving the near-capacity of two-way relay channels with modulation-coded physical-layer network coding," IEEE Trans. Wireless Comm., vol. 14, no. 9, pp. 5225-5239, May 2015.

[30] S. Liew, L. Lu, and S. Zhang, "A primer on physical-layer network coding," Jean Walrand, Series Editor, 2015.

[31] S.-N. Hong and G. Caire, "Structured lattice codes for some two-user gaussian networks with cognition, coordination, and two hops," IEEE Trans. Inf. Theory, vol. 61, pp. 2624-2646, May 2015.

[32] S. H. Park, O. Simeone, O. Sahin, and S. Shamai, "Joint precoding and multivariate backhaul compression for the downlink of cloud radio access networks," IEEE Trans. Signal Processing, vol. 61, no. 22, pp. 5646-5658, Nov. 2013.

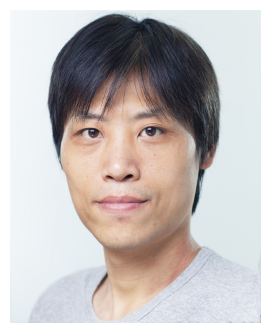

Tao Yang (S'07, M'10) received B.Sc. degree in Electronic Engineering in 2003 from Beijing University of Aero. and Astro. (Beihang University), Beijing, China. He received Master by research and $\mathrm{PhD}$ degrees in Electrical Engineering from the University of New South Wales (UNSW), Sydney, Australia, in 2006 and 2010, respectively. He was an OCE postdoc fellow in Commonwealth Scientific and Industrial Research Organization (CSIRO) ICT centre, Australia. He was with UNSW as a research Fellow. Now he is a lecturer within the Global Big Data Technologies Centre and School of Computing and Communications, University of Technology Sydney. He is now holding an Australian Research Council (ARC) Discovery Early Career Research Award fellowship. His research expertise and interests include physical-layer network coding, multi-user and MIMO, error-control coding, iterative signal processing and network information theory. He has published more than 50 research articles in IEEE journals and conferences. He served as the TPC members of IEEE ICC and WCNC. He was the recipient of Australian Postgraduate Award (APA), NICTA research project award (NRPA) and Supplementary Engineering Award (SEA) from UNSW, and Publication Award from CSIRO ICT centre. 